\title{
Lyapunov Stability of a Fractionally Damped Oscillator with Linear (Anti-)Damping
}

https://doi.org/10.1515/ijnsns-2018-0381

Received December 21, 2018; accepted February 02, 2020

\begin{abstract}
In this paper, we develop a Lyapunov stability framework for fractionally damped mechanical systems. In particular, we study the asymptotic stability of a linear single degree-of-freedom oscillator with viscous and fractional damping. We prove that the total mechanical energy, including the stored energy in the fractional element, is a Lyapunov functional with which one can prove stability of the equilibrium. Furthermore, we develop a strict Lyapunov functional for asymptotic stability, thereby opening the way to a nonlinear stability analysis beyond an eigenvalue analysis. A key result of the paper is a Lyapunov stability condition for systems having negative viscous damping but a sufficient amount of positive fractional damping. This result forms the stepping stone to the study of Hopf bifurcations in fractionally damped mechanical systems. The theory is demonstrated on a stick-slip oscillator with Stribeck friction law leading to an effective negative viscous damping.
\end{abstract}

Keywords: fractional derivative, Lyapunov functional, functional differential equation, Hopf bifurcation, springpot

MSC $^{\circledR}$ (2010). 34K20, 34K37, 37N05

\section{Introduction}

This paper is concerned with the development of a Lyapunov stability framework, including Lyapunov's direct method, for the analysis of stability properties of mechanical systems with fractional damping. The scope of the paper is limited to single degree-of-freedom oscillators with both viscous and fractional damping.

\footnotetext{
*Corresponding author: Matthias Hinze, Institute for Nonlinear Mechanics, University of Stuttgart, Pfaffenwaldring 9, Stuttgart 70569, Germany, E-mail: hinze@inm.uni-stuttgart.de

André Schmidt: E-mail: schmidt@inm.uni-stuttgart.de, Remco I. Leine: E-mail: leine@inm.uni-stuttgart.de, Institute for Nonlinear Mechanics, University of Stuttgart, Stuttgart, Germany
}

The term fractional refers to fractional calculus, which is a mathematical theory dealing with derivatives and integrals of arbitrary (non-integer) order [1, 2] with a variety of applications in science and engineering [3]. Particularly in mechanics, fractional damping may arise through the modeling of mechanical systems with viscoelastic components. Complex rheological models for viscoelastic materials are often described through an array of classical Kelvin or Maxwell elements, inevitably resulting in a model with a large number of parameters. It has been shown that the viscoelastic behavior of complex materials is in many applications well represented by fractional order elements with only a few parameters $[4,5]$. Furthermore, a description using fractional order forcedisplacements relationships may have much better extrapolation properties on long time-scales. The introduction of fractional calculus in mechanics leads to the concept of a springpot element, being a force law reacting linearly on a fractional derivative of its elongation. In a more general setting, springpot elements may also arise through fractional-order control laws [6].

Many problems in industrial applications originate from (dynamic) instability phenomena, e.g. stick-slip vibrations in oilwell drillstrings, flutter of airfoils, shimmy of vehicles and feedback instabilities in control systems. Methods to rigorously prove stability of linear and nonlinear systems are therefore quintessential. The Lyapunov stability framework, which encompasses the method of Lyapunov functions, forms a central element in the research fields Nonlinear Dynamics and Control Theory [7]. The introduction of springpot elements in (controlled) mechanical systems asks for an extension of the Lyapunov stability framework to non-integer order derivatives. A major complication arises through the non-local character of fractional derivatives, i. e. the force in a springpot element depends on the total history of the elongation. A system with springpot elements has therefore an infinite state which asks for the use of Lyapunov functionals instead of Lyapunov functions in Lyapunov's direct method, which are introduced in the theory of functional differential equations (FDEs) [8-12]. Special Lyapunov functionals for FDEs with fractional derivatives are introduced in [13,14], which have been shown to represent the potential energy of an infinite arrangement of springs and 
dashpots [15-17]. The energy expressions for springpots are based on the infinite state or diffusive representation of fractional integrators, which were introduced by Montseny [18], Matignon [19] and have been elaborated by Trigeassou et al. [13, 14, 20, 21]. Beyond that, a lot of work has been done on stability conditions $[22,23]$ and Lyapunov theory [24-26] for fractional differential equations, which cannot directly be used for mechanical systems containing springpots, as the differentiation order is in general irrational for such systems. Furthermore, the Laplace transform method has been used to prove stability of equilibria of mechanical systems containing springpots [27, 28].

The aim of this paper is to give a complete stability analysis of a linear single degree-of-freedom mass-springdashpot-springpot system. The analysis encompasses the following results/tasks:

- The total mechanical energy of the system is derived through the use of the infinite state representation of the springpot element (Section 3).

- The system is put in the form of an FDE and, based on this, definitions of Lyapunov stability and attractivity of fractionally damped mechanical systems are given. Furthermore, a Lyapunov-Krasovskii theorem is presented for this class of systems (Section 4.2).

- It is shown that the total mechanical energy is a Lyapunov functional for the system with positive viscous and fractional damping with which stability of the equilibrium can be proven (Section 4.3). Furthermore, a strict Lyapunov functional for positive viscous and fractional damping is derived which rigorously proves asymptotic stability (Section 4.4). However, this Lyapunov functional fails to give a stability result in the case of anti-damping, in which the viscous damping is negative.

- An extensive eigenvalue analysis is given and, based on that, an expression for the general solution is derived. The eigenvalue analysis reveals that the equilibrium can still be asymptotically stable in the presence of anti-damping (Section 4.5.1).

- A strict Lyapunov functional for the case of antidamping is derived in Section 4.5.2. This leads to a Lyapunov stability condition for systems having negative viscous damping but a sufficient amount of positive fractional damping. Moreover, this result opens the way to study global asymptotic stability of nonlinear systems with fractional damping. The theory is demonstrated on a stick-slip oscillator with Stribeck friction law leading to an effective negative viscous damping (Section 4.5.3).
The scope of the present paper is limited to a single degree-of-freedom oscillator, being of course a first step into the direction of multi degree-of-freedom systems. A brief outlook on how these results can be extended to more degrees of freedom will be given in the conclusion section of the paper.

\section{Fractional calculus and infinite state representation}

We will consider the fractional derivative of Caputo type that is based on the fractional Riemann-Liouville integral, which is defined for an integrable function $x=x(t)$ with $t \geq t_{0}$ and a scalar value $\alpha>0$ as

$$
I_{t_{0}+}^{\alpha} x(t)=\frac{1}{\Gamma(\alpha)} \int_{t_{0}}^{t} x(\tau)(t-\tau)^{\alpha-1} \mathrm{~d} \tau
$$

where $\Gamma(\alpha)$ is the Gamma function. For $\alpha=0$ we set $I_{t_{0}+}^{0} \chi:=x$ and it can be seen directly that the choice $\alpha=1$ leads to the classical integral. In this paper, we will describe this integral operator by the infinite state representation [21]

$$
\left\{\begin{array}{l}
\dot{z}(\omega, t)=-\omega z(\omega, t)+\chi(t), \\
I_{t_{0}+}^{\alpha} \chi(t)=\int_{0}^{\infty} \mu_{\alpha}(\omega) z(\omega, t) \mathrm{d} \omega .
\end{array}\right.
$$

The infinite state $z(\omega, t)$ fulfills the above differential equation $\forall \omega \geq 0$ and the fractional integral is obtained by integrating all contributions $z(\omega, t)$ weighted by the function

$$
\mu_{\alpha}(\omega):=\frac{\sin (\alpha \pi)}{\pi} \omega^{-\alpha}
$$

The equivalence of eqs. (1) and (2) is derived in [15]. The representation (2) is very useful to give a mechanical interpretation of a fractional derivative (Section 3) and to formulate Lyapunov functionals for fractionally damped mechanical systems (Section 4). Using the variation of constants formula, we can formulate eq. (2) as

$$
I_{t_{0}+}^{\alpha} x(t)=\int_{0}^{\infty} \mu_{\alpha}(\omega) \int_{t_{0}}^{t} \mathrm{e}^{-\omega(t-\tau)} \dot{x}(\tau) \mathrm{d} \tau \mathrm{d} \omega .
$$

Finally we introduce the fractional Caputo derivative for an absolutely continuous function $x=x(t)$ and $0<\alpha<1$ as

$$
{ }^{C} D_{t_{0^{+}}}^{\alpha} \chi(t)=I_{t_{0^{+}}}^{1-\alpha} \dot{x}(t) .
$$




\section{Springpot: Mechanical representation and potential energy}

In this section, we will briefly introduce the Caputo springpot (Figure 1) as an abstract mechanical element and discuss its mechanical representation to gain a potential energy expression which may be used for Lyapunov stability considerations of mechanical systems containing springpots. Again, details may be found in [15]. A springpot is defined by its constitutive equation

$$
f(t)={ }^{c} D_{t_{0}+}^{\alpha} q(t)
$$

where $f$ is the force acting on the springpot which results in an elongation $q$ depending on the coefficient $c>0$, initialization time $t_{0}<0$ and differentiation order $\alpha \in(0,1)$. The time interval $\left[t_{0}, 0\right]$ represents the entire significant history of the springpot, i. e. for earlier time-instants $t \leq t_{0}$ we assume $q(t)=0$ and $f(t)=0$.

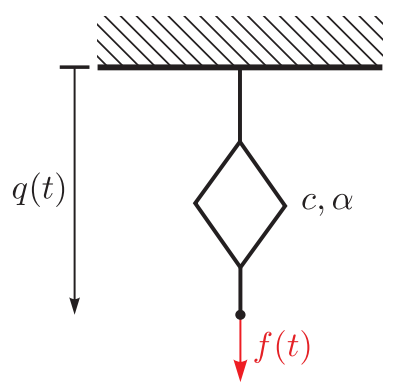

Figure 1: Force acting on a springpot.

Together with eqs. (2) and (5) we derive the infinite state representation of a Caputo springpot as

$$
\left\{\begin{array}{l}
\dot{y}(\omega, t)=-\omega y(\omega, t)+\dot{q}(t), \quad y\left(\omega, t_{0}\right)=0 \\
f(t)=c \int_{0}^{\infty} \mu_{1-\alpha}(\omega) y(\omega, t) \mathrm{d} \omega .
\end{array}\right.
$$

The infinite state $y$ in eq. (7) may, similar as in eq. (4), be expressed by the variation-of-constants formula

$$
y(\omega, t)=\int_{t_{0}}^{t} \mathrm{e}^{-\omega(t-\tau)} \dot{q}(\tau) \mathrm{d} \tau=\int_{t_{0}-t}^{0} \mathrm{e}^{\omega s} \dot{q}(t+s) \mathrm{d} s,
$$

which will be useful for stability considerations later on. The above representation leads to a mechanical analogue model of a springpot, which is a parallel arrangement of an infinite number of Maxwell elements (Figure 2) as in

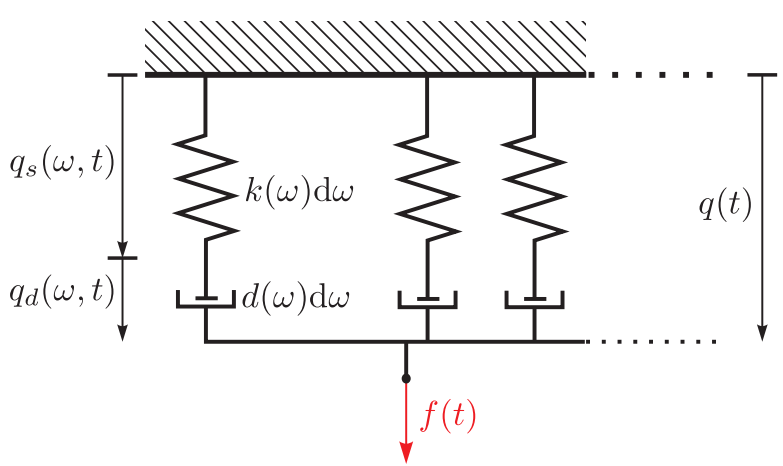

Figure 2: Schematic mechanical representation of a Caputo springpot.

[16], where the forces $g(\omega, t) \mathrm{d} \omega$ of the Maxwell elements are integrated to the resulting force

$$
f(t)=\int_{0}^{\infty} g(\omega, t) \mathrm{d} \omega
$$

on the system. The springs of the Maxwell elements are characterized by their elongation $q_{s}(\omega, t)$ and spring constant $k(\omega) \mathrm{d} \omega$ and the dashpots by the elongation $q_{d}(\omega, t)$ and constant $d(\omega) \mathrm{d} \omega$ such that the elongation of the system $q(t)$ appears as

$$
q(t)=q_{s}(\omega, t)+q_{d}(\omega, t) \quad \forall \omega \geq 0
$$

with the incremental internal force

$$
g(\omega, t) \mathrm{d} \omega=k(\omega) \mathrm{d} \omega q_{s}(\omega, t)=d(\omega) \mathrm{d} \omega \dot{q}_{d}(\omega, t) .
$$

Differentiation of eq. (10) and substitution of eq. (11) leads to

$$
\dot{q}(t)=\frac{\dot{g}(\omega, t)}{k(\omega)}+\frac{g(\omega, t)}{d(\omega)} .
$$

Comparison of eqs. (9) and (12) to (7) results in the identification

$$
\begin{aligned}
g(\omega, t) & =c \mu_{1-\alpha}(\omega) y(\omega, t), \quad k(\omega)=c \mu_{1-\alpha}(\omega), \\
d(\omega) & =\frac{c \mu_{1-\alpha}(\omega)}{\omega}, \quad \omega=\frac{k(\omega)}{d(\omega)} .
\end{aligned}
$$

Furthermore, we obtain an interpretation of the infinite state $y$ of the Caputo springpot as

$$
y(\omega, t)=\frac{g(\omega, t)}{c \mu_{1-\alpha}(\omega)}=\frac{g(\omega, t)}{k(\omega)}=q_{s}(\omega, t) .
$$

Finally, we consider the energy of the mechanical equivalent system which is the potential energy stored in the springs of the Maxwell elements, i. e. 


$$
E(t)=\frac{1}{2} \int_{0}^{\infty} k(\omega) q_{S}^{2}(\omega, t) \mathrm{d} \omega
$$

which can be reformulated with eqs. (13) and (14) as

$$
E(t)=\frac{c}{2} \int_{0}^{\infty} \mu_{1-\alpha}(\omega) y^{2}(\omega, t) \mathrm{d} \omega
$$

\section{Stability}

\subsection{Introduction}

The mechanical representation and the potential energy expressed in terms of the infinite state $y$ were derived more detailed in [15]. The energy expression in eq. (16) was used to prove stability of the equilibrium of a mass-springspringpot system. In the following we want to extend this approach to consider stability of the same system when linear (anti-)damping is introduced, i.e. we regard the system (Figure 3)

$$
m \ddot{q}(t)=-d \dot{q}(t)-c^{C} D_{t_{0}+}^{\alpha} q(t)-k q(t), \quad t \geq 0
$$

with mass $m$, elongation $q(t)$, spring coefficient $k$, springpot coefficient $c$, damping coefficient $d$ and differentiation order $\alpha \in(0,1)$ and given initial functions $\varphi_{1}, \varphi_{2} \in C B((-\infty, 0] ; \mathbb{R})$ such that

$$
\begin{array}{ll}
q(t)=\varphi_{1}(t), & t \leq 0, \\
\dot{q}(t)=\varphi_{2}(t), & t \leq 0,
\end{array}
$$

where $\varphi_{i}(t)=0$ for $t \leq t_{0}, i=1,2$. Again, with the help of eqs. (7) and (8) we reformulate eq. (17) as an FDE

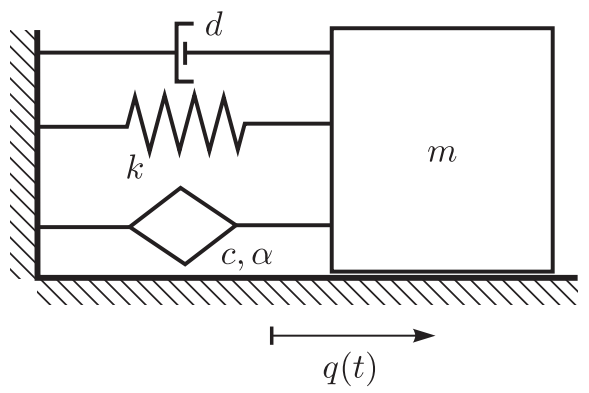

Figure 3: Mass-spring-dashpot-springpot system.

$$
\left\{\begin{aligned}
\dot{q}(t)= & v(t) \\
\dot{v}(t)= & -\frac{k}{m} q(t)-\frac{d}{m} \dot{q}(t) \\
& -\frac{c}{m} \int_{t_{0}-t}^{0} \int_{0}^{\infty} \mu_{1-\alpha}(\omega) \mathrm{e}^{\omega s} \mathrm{~d} \omega v_{t}(s) \mathrm{d} s,
\end{aligned}\right.
$$

where

$$
v_{t}(s)=v(t+s), \quad s \in(-\infty, 0]
$$

and use the associated stability theory. For the cases $d>0$ (damping) and $d<0$ (anti-damping) we use different methods to prove Lyapunov stability of the equilibrium of eq. (17).

\subsection{Theoretical background}

The system (19) is a representative of an FDE of the form

$$
\dot{x}(t)=f\left(t, x_{t}\right), \quad t \geq t_{1} \geq 0
$$

with initial time $t_{1}$ and a map

$$
f: S \rightarrow \mathbb{R}^{n}
$$

defined on

$$
\begin{aligned}
S & =[0, \infty) \times Q_{H}, \\
Q_{H} & :=\left\{\varphi \in C B\left((-\infty, 0] ; \mathbb{R}^{n}\right) \mid\|\varphi\|_{\infty}<H\right\}, H>0,
\end{aligned}
$$

i. e. the function $f$ acts in its second argument on the space $C B\left((-\infty, 0] ; \mathbb{R}^{n}\right)$ of continuous and bounded functions defined on the negative half space together with the norm

$$
\|\varphi\|_{\infty}=\sup _{s \in(-\infty, 0]}\|\varphi(s)\|_{2}
$$

where $\|.\|_{2}$ is the Euclidean norm. Furthermore, let

$$
\begin{gathered}
x \in C\left((-\infty, T) ; \mathbb{R}^{n}\right), \quad T>t_{1}, \\
x_{t}(s)=x(t+s), \quad s \in(-\infty, 0],
\end{gathered}
$$

such that

$$
x_{t} \in C B\left((-\infty, 0] ; \mathbb{R}^{n}\right)
$$

We assume $f$ locally Lipschitzian in $Q_{H}$ which ensures local existence and uniqueness of a solution $x\left(t_{1}, \varphi\right)$ of eq. (21) for a given initial function $\varphi$ and initial time $t_{1}$ $[9,11]$. Moreover, let $f(t, 0)=0 \forall t \geq t_{1}$, such that the trivial solution $x\left(t_{1}, \varphi\right)(t)=0$ is an equilibrium of the 
system. In the following theorem, we formulate sufficient conditions for (asymptotic) stability of the trivial solution of eq. (21) by a Lyapunov theorem adapted to the space $C B\left((-\infty, 0] ; \mathbb{R}^{n}\right)$ as in $[8,9,11]$. We need the following definitions.

Definition 4.1. A solution of eq. (21) with initial function $\varphi \in C B\left((-\infty, 0] ; \mathbb{R}^{n}\right)$ and initial time $t_{1} \geq 0$ is a function $x\left(t_{1}, \varphi\right)$ defined and continuous on an interval $(-\infty, T)$, $T>t_{1}$, such that $x_{t}\left(t_{1}, \varphi\right) \in S$ for $t \in\left[t_{1}, T\right), x_{t_{1}}\left(t_{1}, \varphi\right)=\varphi$ and $x\left(t_{1}, \varphi\right)(t)$ satisfies eq. (21) for $t \in\left[t_{1}, T\right)$.

Definition 4.2 (Stability). The trivial solution $x\left(t_{1}, \varphi\right)(t)=0$ of eq. (21) together with (23), (25), initial function $\varphi$ and initial time $t_{1}$ is called

(a) stable, if for all $\epsilon \in(0, H]$ there exists a $\delta=\delta\left(t_{1}, \epsilon\right)>0$ such that $\left\|x\left(t_{1}, \varphi\right)(t)\right\|_{2}<\epsilon$ for $t \geq t_{1}$ if $\varphi \in Q_{\delta}$.

(b) asymptotically stable, if it is stable and for all $t_{1}$ there exists a $\delta=\delta\left(t_{1}\right)>0$ such that $\lim _{t \rightarrow \infty}\left\|x\left(t_{1}, \varphi\right)(t)\right\|_{2}=0$ if $\varphi \in Q_{\delta}$.

Theorem 4.3 (Lyapunov-Krasovskii $[9,11])$. Let $f: S \rightarrow \mathbb{R}^{n}$ such that $f(t, 0)=0 \forall t \geq t_{1}$ and denote $u_{i}:[0, \infty) \rightarrow \mathbb{R}$, $i=1,2$ some scalar, continuous, non-decreasing functions such that $u_{i}(0)=0$ and $u_{i}(r)>0$ for $r>0$. Let there exist $a$ continuous functional $V:[0, \infty) \times Q_{H} \rightarrow \mathbb{R}$ such that

$$
\begin{gathered}
u_{1}\left(\|\varphi(0)\|_{2}\right) \leq V(t, \varphi), \\
V(t, 0)=0, \quad \dot{V}\left(t, x_{t}\right) \leq 0,
\end{gathered}
$$

then the trivial solution of eq. (21) is stable.

Furthermore, if the additional assumptions

$$
\begin{gathered}
\exists L>0:\|f(t, \varphi)\|_{2}<L, t \geq t_{1}, \varphi \in Q_{H}, \\
\dot{V}\left(t, x_{t}\right) \leq-u_{2}\left(\left\|x\left(t_{1}, \varphi\right)(t)\right\|_{2}\right)
\end{gathered}
$$

are satisfied, then the trivial solution is asymptotically stable.

Remark 4.4. As $f$ is assumed locally Lipschitzian in $Q_{H}$, condition (28) is fulfilled, if the Lipschitz constant $L$ is independent of time $t \geq t_{1}$.

\subsection{Undamped Case}

First, we consider eq. (17) for the case $d=0$ and use the total mechanical energy

$$
\begin{aligned}
V_{1}\left(t, q_{t}, v_{t}\right)= & \frac{1}{2} m v_{t}^{2}(0)+\frac{1}{2} k q_{t}^{2}(0) \\
& +\frac{1}{2} c \int_{0}^{\infty} \mu_{1-\alpha}(\omega) y^{2}(\omega, t) \mathrm{d} \omega
\end{aligned}
$$

as a Lyapunov functional as in [15], where we use a simplified notation in terms of the infinite state $y$ and keep in mind the formulation in eq. (8) or

$$
y(\omega, t)=\int_{t_{0}-t}^{0} \mathrm{e}^{\omega s} v_{t}(s) \mathrm{d} s, \quad \omega \geq 0 .
$$

We prove stability of the trivial solution with the help of Theorem 4.3. It is obvious, that inequality (26) holds for $V_{1}$. Furthermore, as

$$
\begin{aligned}
\dot{V}_{1}\left(t, q_{t}, v_{t}\right)= & k q_{t}(0) \dot{q}_{t}(0)+m v_{t}(0) \dot{v}_{t}(0) \\
& +c \int_{0}^{\infty} \mu_{1-\alpha}(\omega) y(\omega, t) \dot{y}(\omega, t) \mathrm{d} \omega \\
= & v_{t}(0)\left(m \ddot{q}_{t}(0)+k q_{t}(0)\right. \\
& \left.+c \int_{0}^{\infty} \mu_{1-\alpha}(\omega) y(\omega, t) \mathrm{d} \omega\right) \\
& -c \int_{0}^{\infty} \omega \mu_{1-\alpha}(\omega) y^{2}(\omega, t) \mathrm{d} \omega \\
= & -c \int_{0}^{\infty} \omega \mu_{1-\alpha}(\omega) y^{2}(\omega, t) \mathrm{d} \omega \leq 0,
\end{aligned}
$$

inequality (27) is fulfilled, such that the trivial solution is stable.

\subsection{Linear damping}

Using the energy functional $V_{1}$ in the case $d>0$ again leads to a non-positive rate of $V_{1}$

$$
\dot{V}_{1}=-d v^{2}(t)-c \int_{0}^{\infty} \omega \mu_{1-\alpha}(\omega) y^{2}(\omega, t) \mathrm{d} \omega \leq 0,
$$

which only proves stability of the equilibrium. However, we could find an augmented candidate Lyapunov functional which contains the potential energy term (16) to prove asymptotic stability with the help of Theorem 4.3.

Proposition 4.5. The trivial solution of the damped massspring-springpot system (17) (m, $d, k, c>0, \alpha \in[0,1])$ is asymptotically stable.

Proof. We introduce a Lyapunov functional as in Theorem 4.3 defined on $S$ using the simplified notation in terms of the infinite state $y$ as in eq. (31). Furthermore, we introduce another infinite state $Y=Y(\omega, t)$ which satisfies 


$$
\dot{Y}(\omega, t)=-\omega Y(\omega, t)+q(t), \quad Y\left(\omega, t_{0}\right)=0 .
$$

Similar to eq. (31), this may be reformulated as

$$
Y(\omega, t)=\int_{t_{0}-t}^{0} \mathrm{e}^{\omega s} q_{t}(s) \mathrm{d} s, \quad \omega \geq 0
$$

and differentiating eq. (8) and comparing to eqs. (31) and (34) we obtain

$$
y(\omega, t)=\dot{Y}(\omega, t)=-\omega Y(\omega, t)+q(t) .
$$

Hence, we formulate the Lyapunov functional

$$
\begin{aligned}
V_{2}\left(t, q_{t}, v_{t}\right)= & \frac{1}{2} m v_{t}^{2}(0)+\frac{1}{2} k q_{t}^{2}(0)+\frac{d^{2}}{4 m} q_{t}^{2}(0) \\
& +\frac{1}{2} d q_{t}(0) v_{t}(0) \\
& +\frac{1}{2} c \int_{0}^{\infty} \mu_{1-\alpha}(\omega) y^{2}(\omega, t) \mathrm{d} \omega \\
& +\frac{c d}{4 m} \int_{0}^{\infty} \mu_{1-\alpha}(\omega) \omega Y^{2}(\omega, t) \mathrm{d} \omega
\end{aligned}
$$

and check the conditions in Theorem 4.3. For (26) we can estimate

$$
\begin{aligned}
V_{2}\left(t, q_{t}, v_{t}\right) \geq & \frac{1}{2} m v_{t}^{2}(0)+\frac{1}{2} k q_{t}^{2}(0) \\
& +\frac{d^{2}}{4 m} q_{t}^{2}(0)+\frac{1}{2} d q_{t}(0) v_{t}(0) \\
= & \frac{1}{4} m v_{t}^{2}(0)+\frac{1}{2} k q_{t}^{2}(0) \\
& +\left(\frac{d}{2 \sqrt{m}} q_{t}(0)+\frac{\sqrt{m}}{2} v_{t}(0)\right)^{2} \\
& \geq \frac{1}{4} m v_{t}^{2}(0)+\frac{1}{2} k q_{t}^{2}(0) .
\end{aligned}
$$

Moreover, we compute the rate of $V_{2}$ along solution curves

$$
\begin{aligned}
\dot{V}_{2} & =m v_{t}(0) \dot{v}_{t}(0)+k q_{t}(0) \dot{q}_{t}(0)+\frac{d}{2} \dot{q}_{t}(0) v_{t}(0) \\
& +\frac{d}{2} q_{t}(0) \dot{v}_{t}(0)+\frac{d^{2}}{2 m} q_{t}(0) \dot{q}_{t}(0) \\
& +c \int_{0}^{\infty} \mu_{1-\alpha}(\omega) y(\omega, t) \dot{y}(\omega, t) \mathrm{d} \omega \\
& +\frac{c d}{2 m} \int_{0}^{\infty} \mu_{1-\alpha}(\omega) \omega Y(\omega, t) \dot{Y}(\omega, t) \mathrm{d} \omega \\
= & -d v_{t}^{2}(0)-c v_{t}(0) \int_{0}^{\infty} \mu_{1-\alpha}(\omega) y(\omega, t) \mathrm{d} \omega \\
& +\frac{d}{2} v_{t}^{2}(0)+\frac{d}{2} q_{t}(0)\left[-\frac{k}{m} q_{t}(0)-\frac{d}{m} v_{t}(0)\right. \\
& \left.-\frac{c}{m} \int_{0}^{\infty} \mu_{1-\alpha}(\omega) y(\omega, t) \mathrm{d} \omega\right]+\frac{d^{2}}{2 m} q_{t}(0) v_{t}(0)
\end{aligned}
$$

$$
\begin{aligned}
& +c \int_{0}^{\infty} \mu_{1-\alpha}(\omega) y(\omega, t)\left(v_{t}(0)-\omega y(\omega, t)\right) \mathrm{d} \omega \\
& +\frac{c d}{2 m} \int_{0}^{\infty} \mu_{1-\alpha}(\omega)\left(q_{t}(0)-y(\omega, t)\right) y(\omega, t) \mathrm{d} \omega \\
= & -\frac{d}{2} v_{t}^{2}(0)-\frac{k d}{2 m} q_{t}^{2}(0) \\
& -c \int_{0}^{\infty} \mu_{1-\alpha}(\omega)\left(\omega+\frac{d}{2 m}\right) y^{2}(\omega, t) \mathrm{d} \omega \\
\leq & -\frac{d}{2} v^{2}(t)-\frac{k d}{2 m} q^{2}(t)
\end{aligned}
$$

which proves inequality (29). Finally, we still have to check condition (28), which is non-trivial only for the last addend of the right-hand side of the second equation in (19). For this term we have to split the interval of integration in two parts which yields for $(1, \infty)$

$$
\begin{aligned}
\mid \int_{1}^{\infty} & \mu_{1-\alpha}(\omega) \int_{t_{0}-t}^{0} \mathrm{e}^{\omega s} v_{t}(s) \mathrm{d} s \mathrm{~d} \omega \mid \\
& \leq \int_{1}^{\infty} \mu_{1-\alpha}(\omega) \int_{-\infty}^{0} \mathrm{e}^{\omega s} \mathrm{~d} s \mathrm{~d} \omega\left\|v_{t}\right\|_{\infty} \\
& =\frac{\sin (\alpha \pi)}{\pi} \int_{1}^{\infty} \omega^{\alpha-2} \mathrm{~d} \omega\left\|v_{t}\right\|_{\infty} \\
& =\frac{\sin (\alpha \pi)}{(1-\alpha) \pi}\left\|v_{t}\right\|_{\infty} .
\end{aligned}
$$

For the integration over $(0,1)$, we achieve together with eq. (36)

$$
\begin{aligned}
& \left|\int_{0}^{1} \mu_{1-\alpha}(\omega) \int_{t_{0}-t}^{0} \mathrm{e}^{\omega s} \dot{q}_{t}(s) \mathrm{d} s \mathrm{~d} \omega\right| \\
& \quad \leq \int_{0}^{1} \mu_{1-\alpha}(\omega)\left|q(t)-\omega \int_{t_{0}-t}^{0} \mathrm{e}^{\omega s} q_{t}(s) \mathrm{d} s\right| \mathrm{d} \omega \\
& \quad \leq \int_{0}^{1} \mu_{1-\alpha}(\omega)\left(\left\|q_{t}\right\|_{\infty}+\omega \int_{-\infty}^{0} \mathrm{e}^{\omega s} \mathrm{~d} s\left\|q_{t}\right\|_{\infty}\right) \mathrm{d} \omega \\
& \quad=2 \int_{0}^{1} \mu_{1-\alpha}(\omega) \mathrm{d} \omega\left\|q_{t}\right\|_{\infty} \\
& \quad=2 \frac{\sin (\alpha \pi)}{\alpha \pi}\left\|q_{t}\right\|_{\infty} .
\end{aligned}
$$

This completes the proof.

\subsection{Linear anti-damping}

For the case $d<0$, whose physical interpretation is explained and motivated in Section 4.5.3, we expect the equilibrium of eq. (17) to remain stable only for certain values of $d$ and it appears to be much more difficult to find Lyapunov functionals that yield stability criteria. Therefore, we start with the Laplace transform method to find sufficient conditions for stability and try to find 
similar conditions with the help of a Lyapunov functional. Finally, we compare the results.

\subsubsection{Laplace transform method}

Before we introduce the Laplace transform of eq. (17), we derive the Laplace transform of the fractional derivative. Therefore, consider the Laplace transform of (7)

$$
\begin{aligned}
& s \mathcal{L}\{y(\omega, t)\}(s)-y(\omega, 0) \\
& =-\omega \mathcal{L}\{y(\omega, t)\}(s)+s Q(s)-q_{0}, \\
& \mathcal{L}\left\{{ }^{C} D_{t_{0}+}^{\alpha} q(t)\right\}(s)=\int_{0}^{\infty} \mu_{1-\alpha}(\omega) \mathcal{L}\{y(\omega, t)\}(s) \mathrm{d} \omega .
\end{aligned}
$$

with Laplace transform $Q(s)$ of $q(t)$ and initial value $q(0)=q_{0}$. Substitution of the first equation of (39) in the second results in

$$
\begin{aligned}
\mathcal{L} & \left\{{ }^{C} D_{t_{0}+}^{\alpha} q(t)\right\}(s) \\
& =\int_{0}^{\infty} \mu_{1-\alpha}(\omega) \frac{s Q(s)-q_{0}+y(\omega, 0)}{\omega+s} \mathrm{~d} \omega .
\end{aligned}
$$

We reformulate (40) with the help of the following relation.

\section{Proposition 4.6.}

$$
\int_{0}^{\infty} \frac{\mu_{\alpha}(\omega)}{\omega+s} \mathrm{~d} \omega=s^{-\alpha}, \quad s \in \mathbb{C} \backslash \mathbb{R}^{-}, \alpha \in(0,1)
$$

Proof. Due to the relation for the Laplace transform of $\mathrm{e}^{-\omega t}$

$$
\begin{aligned}
\mathcal{L}\left\{\mathrm{e}^{-\omega t}\right\}(s) & =\int_{0}^{\infty} \mathrm{e}^{-\omega t} \mathrm{e}^{-s t} \mathrm{~d} t=\int_{0}^{\infty} \mathrm{e}^{-(\omega+s) t} \mathrm{~d} t \\
& =\left[-\frac{1}{\omega+s} \mathrm{e}^{-(\omega+s) t}\right]_{0}^{\infty}=\frac{1}{\omega+s}
\end{aligned}
$$

we obtain eq. (41) using the formula

$$
\Gamma(\alpha) \Gamma(1-\alpha)=\frac{\pi}{\sin (\alpha \pi)}
$$

and Fubini's Theorem as

$$
\begin{aligned}
\int_{0}^{\infty} \frac{\mu_{\alpha}(\omega)}{\omega+s} \mathrm{~d} \omega & =\frac{\sin (\alpha \pi)}{\pi} \int_{0}^{\infty} \omega^{-\alpha} \int_{0}^{\infty} \mathrm{e}^{-(\omega+s) t} \mathrm{~d} t \mathrm{~d} \omega \\
& =\frac{\sin (\alpha \pi)}{\pi} \int_{0}^{\infty} \mathrm{e}^{-s t} \int_{0}^{\infty} \omega^{-\alpha} \mathrm{e}^{-\omega t} \mathrm{~d} \omega \mathrm{d} t \\
& =\frac{\sin (\alpha \pi)}{\pi} \int_{0}^{\infty} \mathrm{e}^{-s t} \Gamma(1-\alpha) t^{\alpha-1} \mathrm{~d} t \\
& =\frac{\sin (\alpha \pi)}{\pi} \Gamma(1-\alpha) \Gamma(\alpha) s^{-\alpha}=s^{-\alpha} .
\end{aligned}
$$

This leads to the Laplace transform of the fractional derivative

$$
\begin{aligned}
\mathcal{L} & \left\{{ }^{C} D_{t_{0}+}^{\alpha} q(t)\right\}(s) \\
& =s^{\alpha} Q(s)-s^{\alpha-1} q_{0}+\int_{0}^{\infty} \mu_{1-\alpha}(\omega) \frac{y(\omega, 0)}{\omega+s} \mathrm{~d} \omega .
\end{aligned}
$$

Hence, we obtain the Laplace transform of eq. (17) as

$$
\begin{aligned}
m & \left(s^{2} Q(s)-s q_{0}-v_{0}\right) \\
= & -k Q(s)-c\left(s^{\alpha} Q(s)-s^{\alpha-1} q_{0}\right. \\
& \left.+\int_{0}^{\infty} \mu_{1-\alpha}(\omega) \frac{y(\omega, 0)}{\omega+s} \mathrm{~d} \omega\right) \\
& -d\left(s Q(s)-q_{0}\right)
\end{aligned}
$$

with Laplace transform $Q(s)$ of $q(t)$ and initial values $q(0)=q_{0}, \dot{q}(0)=v_{0}$. Solving equation (43) for $Q(s)$ leads to

$$
\begin{aligned}
Q(s)= & \frac{m\left(s q_{0}+v_{0}\right)}{m s^{2}+d s+c s^{\alpha}+k} \\
& +c \frac{s^{\alpha-1} q_{0}-\int_{0}^{\infty} \mu_{1-\alpha}(\omega) \frac{y(\omega, 0)}{\omega+s} \mathrm{~d} \omega}{m s^{2}+d s+c s^{\alpha}+k} \\
& +\frac{d q_{0}}{m s^{2}+d s+c s^{\alpha}+k} .
\end{aligned}
$$

The inverse Laplace transform may be obtained integrating along a Hankel contour and using the residue theorem, similar as described in [27, 28]. We will accomplish the entire derivation later and see, that, similar to the classical case, stability of the equilibrium depends on the real part of the poles of the right-hand side of eq. (44), i. e. we consider the equation

$$
m s^{2}+d s+c s^{\alpha}+k=0 .
$$

Let $s=r \mathrm{e}^{i \theta}$, we obtain real and imaginary part of eq. (45) as

$$
\begin{aligned}
m r^{2} \cos (2 \theta)+d r \cos (\theta)+c r^{\alpha} \cos (\alpha \theta)+k & =0, \\
m r^{2} \sin (2 \theta)+d r \sin (\theta)+c r^{\alpha} \sin (\alpha \theta) & =0 .
\end{aligned}
$$

From (46) we want to derive conditions, such that the roots of eq. (45) are located in the left-half complex plane. Therefore, we first consider the critical case for stability $\theta= \pm \frac{\pi}{2}$, which turns (46) into

$$
\begin{array}{r}
-m r^{2}+c r^{\alpha} \cos \left(\frac{\alpha \pi}{2}\right)+k=0, \\
d r+c r^{\alpha} \sin \left(\frac{\alpha \pi}{2}\right)=0 .
\end{array}
$$


For fixed $m, c, k$ and $\alpha$ the first equation of (47) has a unique solution $r=r_{*}>0$, which may be inserted in the second equation to compute a critical $d<0$ for stability

$$
d_{\text {crit }}=-c \sin \left(\frac{\alpha \pi}{2}\right) r_{*}^{\alpha-1}
$$

By numerical solution of eq. (47), we obtain the critical negative damping parameter $d_{\text {crit }}$ depending on the value of $\alpha \in(0,1)$ and the parameters $m, c, k$, see Figure 4. The value $\left|d_{\text {crit }}\right|$ is a measure for the damping capability of the springpot. As expected, it holds that $d_{\text {crit }} \rightarrow 0$ for $\alpha \rightarrow 0$, as in this case the springpot degenerates to a spring, which stores energy and $d_{\text {crit }} \rightarrow-c$ for $\alpha \rightarrow 1$, as the springpot becomes a dashpot. The dependency of $d_{\text {crit }}$ on $\alpha$ for $\alpha \in(0,1)$ may change drastically for different parameters and it is quite interesting that $d_{\text {crit }}<-c$ can be achieved for certain values of $\alpha$, i. e. a springpot can induce higher damping than a dashpot with the same coefficient. An example for this phenomenon is given in
Section 4.5.3, where the knowledge (and tuning) of the system parameters $m, k, c$ and $\alpha$ lead to the characterization of a Stribeck friction law. From the critical case for stability we now derive the following inequality conditions on $r$, such that a solution $s=r \mathrm{e}^{i \theta}$ of (45) is located in the left-half complex plane. More specifically, we obtain the following proposition.

Proposition 4.7. Let the inequalities

$$
\begin{gathered}
-m r^{2}+c r^{\alpha} \cos \left(\frac{\alpha \pi}{2}\right)+k \leq 0, \\
d r+c r^{\alpha} \sin \left(\frac{\alpha \pi}{2}\right)>0
\end{gathered}
$$

have a non-empty solution set for $r>0$. Then there exists a pair of complex conjugate roots $s=r \mathrm{e}^{i \theta}, \bar{s}=r \mathrm{e}^{-i \theta}$ of (45) such that $\frac{\pi}{2}<\theta<\frac{\pi}{2-\alpha}$. Furthermore, there exists no solution outside the sectors $\left\{\theta \in\left(\frac{\pi}{2}, \pi\right)\right\}$ and $\left\{\theta \in\left(-\pi,-\frac{\pi}{2}\right)\right\}$.
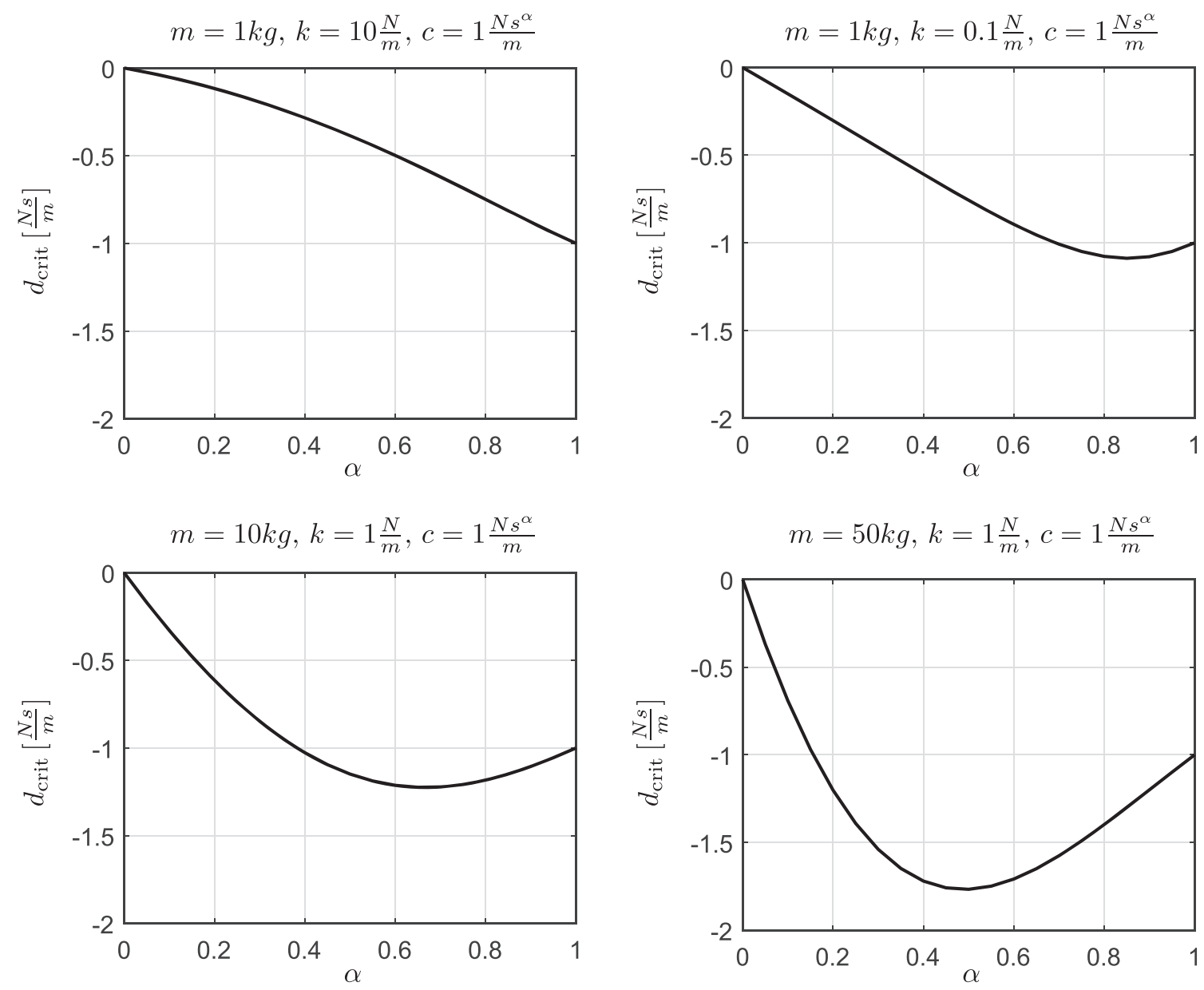

Figure 4: Critical negative damping parameter depending on $\alpha \in(0,1)$ for different parameters. 

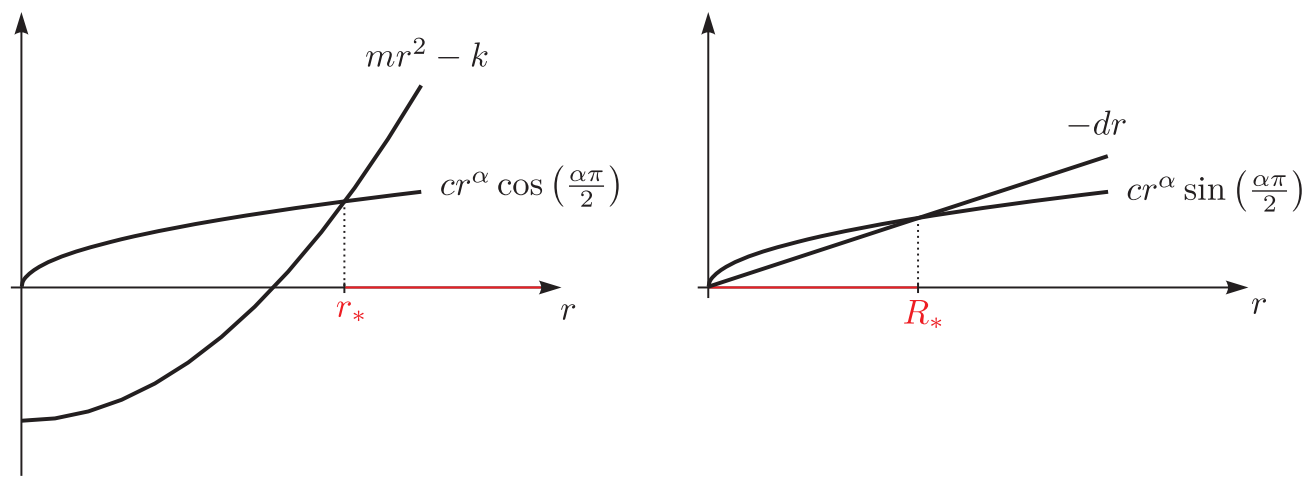

Figure 5: Representation of the solution set of inequalities (49) and (50).

Remark 4.8. To depict the solution set of inequalities (49) and (50), consider Figure 5 below, where $r_{*}<R_{*}$ has to hold.

Proof of Proposition 4.7. From (46) we see that for each root $s=r \mathrm{e}^{\mathrm{i} \theta}$ of equation (45), its complex conjugate $\bar{s}=r \mathrm{e}^{-i \theta}$ is another root. Therefore, we only consider $0 \leq \theta \leq \pi$. We examine the following cases.

- Case 1: $\theta=0$

In this case, eq. (45) degenerates to one equation

$$
m r^{2}+d r+c r^{\alpha}+k=0
$$

Using eqs. (49) and (50), we can estimate

$$
\begin{aligned}
m r^{2} & +d r+c r^{\alpha}+k \\
& >c r^{\alpha}\left(1+\cos \left(\frac{\alpha \pi}{2}\right)-\sin \left(\frac{\alpha \pi}{2}\right)\right)+2 k
\end{aligned}
$$

As the function

$$
h(\alpha):=1+\cos \left(\frac{\alpha \pi}{2}\right)-\sin \left(\frac{\alpha \pi}{2}\right)
$$

fulfills

$$
\begin{aligned}
& h(0)=2, \quad h(1)=0, \\
& h^{\prime}(\alpha)=-\frac{\pi}{2}\left(\sin \left(\frac{\alpha \pi}{2}\right)+\cos \left(\frac{\alpha \pi}{2}\right)\right)<0 \\
& \forall \alpha \in(0,1),
\end{aligned}
$$

we obtain

$$
m r^{2}+d r+c r^{\alpha}+k>0
$$

and there exists no solution of eq. (51).

- Case 2: $\theta=\pi$

The second equation of (46) in this case reads as

$$
\operatorname{cr}^{\alpha} \sin (\alpha \pi)=0,
$$

which has no solution for $\alpha \in(0,1)$ except $r=0$, which does not solve the first equation of (46)

$$
m r^{2}-d r+c r^{\alpha} \cos (\alpha \pi)+k=0 .
$$

- Case 3: $0<\theta<\frac{\pi}{2}$

Multiplying the first equation of (46) by $\cos (\theta)$ and the second by $\sin (\theta)$ sums up as

$$
m r^{2} \cos (\theta)+d r+c r^{\alpha} \cos ((1-\alpha) \theta)+k \cos (\theta)=0 .
$$

The left-hand side of eq. (52) may be estimated with (49) and (50) as

$$
\begin{gathered}
m r^{2} \cos (\theta)+d r+c r^{\alpha} \cos ((1-\alpha) \theta)+k \cos (\theta) \\
>c r^{\alpha}\left(\cos (\theta) \cos \left(\frac{\alpha \pi}{2}\right)+\cos ((1-\alpha) \theta)\right. \\
\left.-\sin \left(\frac{\alpha \pi}{2}\right)\right)+2 k \cos (\theta)>0,
\end{gathered}
$$

because

$$
\cos (\theta) \cos \left(\frac{\alpha \pi}{2}\right)>0
$$

and

$$
\begin{aligned}
& \cos ((1-\alpha) \theta)-\sin \left(\frac{\alpha \pi}{2}\right) \\
& \quad=\cos ((1-\alpha) \theta)-\cos \left((1-\alpha) \frac{\pi}{2}\right)>0 .
\end{aligned}
$$

Hence, there is no solution of eq. (45) for $\theta \in\left(0, \frac{\pi}{2}\right)$ and $\alpha \in(0,1)$.

- Case 4: $\frac{\pi}{2}<\theta<\pi$

Multiplying the second equation of (46) by $\cos (\alpha \theta)$ and subtracting the first equation multiplied by $\sin (\alpha \theta)$ leads to

$$
m r^{2} \sin ((2-\alpha) \theta)+d r \sin ((1-\alpha) \theta)-k \sin (\alpha \theta)=0,
$$


which may be solved for $r>0$ as

$$
\begin{aligned}
r(\theta) & =-\frac{d}{2 m} \frac{\sin ((1-\alpha) \theta)}{\sin ((2-\alpha) \theta)} \\
& +\sqrt{\left(\frac{d}{2 m} \frac{\sin ((1-\alpha) \theta)}{\sin ((2-\alpha) \theta)}\right)^{2}+\frac{k}{m} \frac{\sin (\alpha \theta)}{\sin ((2-\alpha) \theta)}} .
\end{aligned}
$$

Furthermore, multiplying the first equation of (46) by $\sin (2 \theta)$ and subtracting the second equation multiplied by $\cos (2 \theta)$ leads to

$$
d r \sin (\theta)+c r^{\alpha} \sin ((2-\alpha) \theta)+k \sin (2 \theta)=0 .
$$

As the first and the last term on the left-hand side of eq. (54) are negative for $\theta \in\left(\frac{\pi}{2}, \pi\right)$, the second term has to be positive to solve the equation, i. e.

$$
\sin ((2-\alpha) \theta)>0 \Rightarrow \frac{\pi}{2}<\theta<\frac{\pi}{2-\alpha} .
$$

Now, we consider the left-hand side of eq. (54) as a function of $\theta$

$$
\begin{aligned}
g(\theta):= & d r(\theta) \sin (\theta)+c r^{\alpha}(\theta) \sin ((2-\alpha) \theta) \\
& +k \sin (2 \theta), \quad \theta \in\left(\frac{\pi}{2}, \frac{\pi}{2-\alpha}\right)
\end{aligned}
$$

with $r(\theta)$ given by eq. (53). The function $g$ is continuous for $\theta \in\left(\frac{\pi}{2}, \frac{\pi}{2-\alpha}\right)$ and it holds that

$$
\begin{aligned}
g\left(\frac{\pi}{2}\right) & =d r+c r^{\alpha} \sin \left((2-\alpha) \frac{\pi}{2}\right) \\
& =d r+c r^{\alpha} \sin \left(\frac{\alpha \pi}{2}\right)>0
\end{aligned}
$$

as follows from (50). Furthermore, it can be seen from (53), that there exists a constant $C>0$, such that

$$
\lim _{\theta \rightarrow \frac{\pi}{2-\alpha}} r(\theta)=\lim _{\theta \rightarrow \frac{\pi}{2-\alpha}} \frac{C}{\sin ((2-\alpha) \theta)}=\infty,
$$

so that

$$
\begin{aligned}
\lim _{\theta \rightarrow \frac{\pi}{2-\alpha}} g(\theta)= & \lim _{\theta \rightarrow \frac{\pi}{2-\alpha}}\left[d \frac{C}{\sin ((2-\alpha) \theta)} \sin \left(\frac{\pi}{2-\alpha}\right)\right. \\
& +c\left(\frac{C}{\sin ((2-\alpha) \theta)}\right)^{\alpha} \sin ((2-\alpha) \theta) \\
& \left.+k \sin \left(\frac{2 \pi}{2-\alpha}\right)\right]=-\infty .
\end{aligned}
$$

Therefore, there is at least one root of $g$, i.e. one pair of complex conjugate solutions of eq. (45) such that $\theta \in\left(\frac{\pi}{2}, \frac{\pi}{2-\alpha}\right)$.
As we have found conditions for solutions of the characteristic eq. (45) to be in the left-half complex plane, we want to prove asymptotic stability of the trivial solution by inverse Laplace transform using fundamental ideas of complex analysis. Therefore, we reformulate eq. (44) as

$$
\begin{aligned}
Q(s)= & \frac{m s+d+c s^{\alpha-1}}{m s^{2}+d s+c s^{\alpha}+k} q_{0}+\frac{m}{m s^{2}+d s+c s^{\alpha}+k} v_{0} \\
& -\frac{c}{m s^{2}+d s+c s^{\alpha}+k} \int_{0}^{\infty} \mu_{1-\alpha}(\omega) \frac{y_{0}(\omega)}{\omega+s} \mathrm{~d} \omega .
\end{aligned}
$$

Similar as in [27] we consider the function

$$
\Xi(s):=\frac{m s+d+c s^{\alpha-1}}{m s^{2}+d s+c s^{\alpha}+k}
$$

and we compute the inverse Laplace transform $\xi(t)$ of $\Xi(s)=\mathcal{L}\{\xi(t)\}(s)$. As

$$
\xi(0)=\lim _{s \rightarrow \infty} s \Xi(s)=1,
$$

we obtain

$$
\begin{aligned}
\mathcal{L}\{\dot{\xi}(t)\}(s) & =s \Xi(s)-\xi(0)=\frac{m s^{2}+d s+c s^{\alpha}}{m s^{2}+d s+c s^{\alpha}+k}-1 \\
& =-\frac{k}{m s^{2}+d s+c s^{\alpha}+k},
\end{aligned}
$$

which, together with eq. (55) leads to the solution

$$
\begin{aligned}
q(t)= & q_{0} \xi(t)-\frac{m}{k} v_{0} \dot{\xi}(t) \\
& +\frac{c}{k} \int_{0}^{\infty} \mu_{1-\alpha}(\omega) y(\omega, 0) \int_{0}^{t} \mathrm{e}^{-\omega(t-\tau)} \dot{\xi}(\tau) \mathrm{d} \tau \mathrm{d} \omega
\end{aligned}
$$

of eq. (17) and we examine the asymptotic behavior of $q$ from $\xi$ and $\dot{\xi}$. Therefore, we determine the inverse Laplace transform

$$
\xi(t)=\frac{1}{2 \pi i} \int_{\sigma-i \infty}^{\sigma+i \infty} \Xi(s) \mathrm{e}^{s t} \mathrm{~d} s, \quad \operatorname{Re}(\sigma)>0
$$

with the help of the residue theorem

$$
\frac{1}{2 \pi i} \int_{\Sigma} \Xi(s) \mathrm{e}^{s t} \mathrm{~d} s=\sum_{j} \operatorname{Res}\left(\Xi(s) \mathrm{e}^{s t}, s_{j}\right),
$$

where $s_{j}$ are the roots of eq. (45) and the closed curve $\Sigma$ (Figure 6) is split up in six parts, such that

$$
\begin{aligned}
\xi(t)= & \sum_{j} \operatorname{Res}\left(\Xi(s) \mathrm{e}^{s t}, s_{j}\right) \\
& -\frac{1}{2 \pi i} \lim _{\substack{x \rightarrow \infty \\
\varepsilon \rightarrow 0}} \int_{\text {II-VI }} \Xi(s) \mathrm{e}^{s t} \mathrm{~d} s .
\end{aligned}
$$




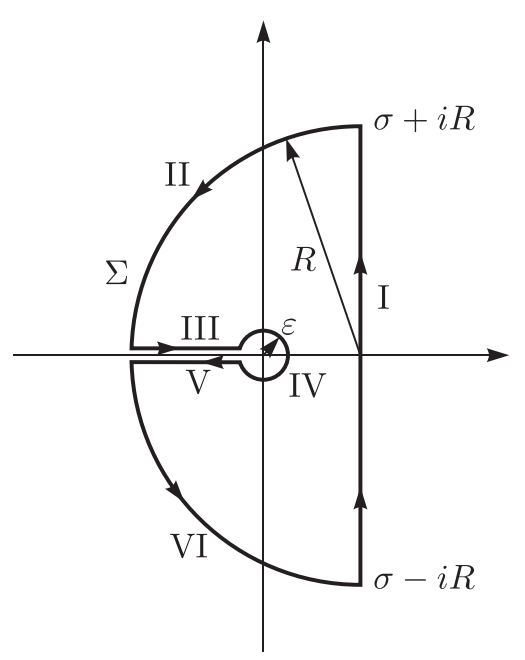

Figure 6: Curve $\Sigma$ used for integration to apply the residue theorem.

First, we compute the residues for a pair of complex conjugate roots $s_{1}, s_{2}=\overline{s_{1}}$ of eq. (45). As $s_{1 / 2}$ are simple poles of $\Xi$, we obtain the residue by derivation of the denominator as

$$
\begin{gathered}
\operatorname{Res}\left(\Xi(s) \mathrm{e}^{s t}, s_{1}\right)+\operatorname{Res}\left(\Xi(s) \mathrm{e}^{s t}, s_{2}\right) \\
=\frac{m s_{1}+d+c s_{1}^{\alpha-1}}{2 m s_{1}+d+c \alpha s_{1}^{\alpha-1}} \mathrm{e}^{s_{1} t} \\
\quad+\frac{m s_{2}+d+c s_{2}^{\alpha-1}}{2 m s_{2}+d+c \alpha s_{2}^{\alpha-1}} \mathrm{e}^{s_{2} t} .
\end{gathered}
$$

As the two addends are conjugate, we obtain with $s_{1}=a+i b=r \mathrm{e}^{i \theta}$

$$
\begin{aligned}
\operatorname{Res} & \left(\Xi(s) \mathrm{e}^{s t}, s_{1}\right)+\operatorname{Res}\left(\Xi(s) \mathrm{e}^{s t}, s_{2}\right) \\
& =2 \operatorname{Re}\left(\frac{m s_{1}+d+c s_{1}^{\alpha-1}}{2 m s_{1}+d+c \alpha s_{1}^{\alpha-1}} \mathrm{e}^{s_{1} t}\right) \\
& =2 \mathrm{e}^{a t} \cos (b t) \frac{f_{1}(r, \theta)}{f_{3}(r, \theta)}+2 \mathrm{e}^{a t} \sin (b t) \frac{f_{2}(r, \theta)}{f_{3}(r, \theta)} .
\end{aligned}
$$

with

$$
\begin{aligned}
f_{1}(r, \theta)= & 2 m^{2} r^{2}+d^{2}+3 m r d \cos (\theta) \\
& +(1+\alpha) c d r^{\alpha-1} \cos ((1-\alpha) \theta) \\
& +(2+\alpha) m c r^{\alpha} \cos ((2-\alpha) \theta)+c^{2} \alpha r^{2(\alpha-1)}, \\
f_{2}(r, \theta)= & (2-\alpha) m c r^{\alpha} \sin ((2-\alpha) \theta)+m d r \sin (\theta) \\
& +(1-\alpha) c d r^{\alpha-1} \sin ((1-\alpha) \theta), \\
f_{3}(r, \theta)= & 4 m^{2} r^{2}+d^{2}+4 m d r \cos (\theta) \\
& +2 c d \alpha r^{\alpha-1} \cos ((1-\alpha) \theta) \\
& +4 m c \alpha r^{\alpha} \cos ((2-\alpha) \theta)+c^{2} \alpha^{2} r^{2(\alpha-1)} .
\end{aligned}
$$

We continue considering the contribution of the integral along the paths II - VI to the value of $\xi$. There is no contribution of II, because

$$
\begin{aligned}
& \left|\frac{1}{2 \pi i} \int_{\text {II }} \Xi(s) \mathrm{e}^{s t} \mathrm{~d} s\right| \\
& =\left|\frac{1}{2 \pi i} \int_{\frac{\pi}{2}}^{\pi} \Xi\left(\sigma+R \mathrm{e}^{i \phi}\right) \mathrm{e}^{\sigma t} \mathrm{e}^{R \cos (\phi) t} \mathrm{e}^{i R \sin (\phi) t} i R \mathrm{e}^{i \phi} \mathrm{d} \phi\right| \\
& \leq \frac{1}{2 \pi} C_{1}(t) \mathrm{e}^{-C_{2} R t} R \cdot \frac{\pi}{2} R \underset{R \rightarrow \infty}{\longrightarrow} 0,
\end{aligned}
$$

with $C_{1}, C_{2}>0$ as $\Xi(s) \rightarrow 0$ for $s \rightarrow \infty$ and $\cos (\phi)<0$ for $\phi \in\left(\frac{\pi}{2}, \pi\right)$. The same argumentation holds for path VI. For path IV, we obtain

$$
\begin{aligned}
\frac{1}{2 \pi i} \int_{\mathrm{IV}} \Xi(s) \mathrm{e}^{s t} \mathrm{~d} s \\
=-\frac{1}{2 \pi i} \int_{-\pi}^{\pi} \Xi\left(\varepsilon \mathrm{e}^{i \phi}\right) \mathrm{e}^{\varepsilon t(\cos (\phi)+i \sin (\phi))} i \varepsilon \mathrm{e}^{i \phi} \mathrm{d} \phi \\
\quad \underset{\varepsilon \rightarrow 0}{\longrightarrow} 0,
\end{aligned}
$$

as $s \Xi(s) \rightarrow 0$ for $s \rightarrow 0$. Finally, for III and $\mathrm{V}$ we obtain a contribution

$$
\begin{aligned}
\frac{1}{2 \pi i} & \int_{\text {III, },} \Xi(s) \mathrm{e}^{s t} \mathrm{~d} s \\
& =\frac{1}{2 \pi i} \int_{0}^{\infty}\left(\Xi\left(\omega \mathrm{e}^{i \pi}\right)-\Xi\left(\omega \mathrm{e}^{-i \pi}\right)\right) \mathrm{e}^{-\omega t} \mathrm{~d} \omega \\
& =\frac{1}{\pi} \int_{0}^{\infty} \operatorname{Im}\left(\Xi\left(\omega \mathrm{e}^{i \pi}\right)\right) \mathrm{e}^{-\omega t} \mathrm{~d} \omega
\end{aligned}
$$

with

$$
\begin{aligned}
& \frac{1}{\pi} \operatorname{Im}\left(\Xi\left(\omega \mathrm{e}^{i \pi}\right)\right) \\
& =-\frac{1}{\pi} \frac{k c \omega^{\alpha-1} \sin (\alpha \pi)}{\left(m \omega^{2}-d \omega+k\right)^{2}+2 c \omega^{\alpha} \cos (\alpha \pi)\left(m \omega^{2}-d \omega+k\right)+c^{2} \omega^{2 \alpha}} \\
& =-\frac{\mu_{1-\alpha}(\omega) k c}{\left(m \omega^{2}-d \omega+k\right)^{2}+2 c \omega^{\alpha} \cos (\alpha \pi)\left(m \omega^{2}-d \omega+k\right)+c^{2} \omega^{2 \alpha}} .
\end{aligned}
$$

This leads us to the inverse Laplace transform of $\Xi$

$$
\begin{aligned}
\xi(t)= & \sum_{j \text { odd }}\left(2 \mathrm{e}^{a_{j} t} \cos \left(b_{j} t\right) \frac{f_{1}\left(r_{j}, \theta_{j}\right)}{f_{3}\left(r_{j}, \theta_{j}\right)}\right. \\
& \left.+2 \mathrm{e}^{a_{j} t} \sin \left(b_{j} t\right) \frac{f_{2}\left(r_{j}, \theta_{j}\right)}{f_{3}\left(r_{j}, \theta_{j}\right)}\right) \\
& +\int_{0}^{\infty} \mu_{1-\alpha}(\omega) Z(\omega) \mathrm{e}^{-\omega t} \mathrm{~d} \omega
\end{aligned}
$$

for roots $s_{j / j+1}=a_{j} \pm i b_{j}=r_{j} \mathrm{e}^{ \pm i \theta_{j}}$ of eq. (45) where

$$
Z(\omega)=\frac{k c}{\left(m \omega^{2}-d \omega+k\right)^{2}+2 c \omega^{\alpha} \cos (\alpha \pi)\left(m \omega^{2}-d \omega+k\right)+c^{2} \omega^{2 \alpha}} .
$$


The asymptotic behavior of $\xi$ is determined by the exponential functions in the first addends of eq. (60), which decay, as $a_{j}<0 \forall j$, if the inequalities (49) and (50) have a non-empty solution set. Furthermore, the asymptotic behavior of the last term in eq. (60) may be estimated as follows. It holds that

$$
\begin{aligned}
\left(m \omega^{2}\right. & -d \omega+k)^{2}+2 c \omega^{\alpha} \cos (\alpha \pi)\left(m \omega^{2}-d \omega+k\right)+c^{2} \omega^{2 \alpha} \\
& >\left(m \omega^{2}-d \omega+k-c \omega^{\alpha}\right)^{2} \geq 0 .
\end{aligned}
$$

Hence, $Z$ is continuous and bounded in $[0, \infty)$ and by the mean value theorem, there exists $C_{3}>0$ such that

$$
\begin{aligned}
\int_{0}^{\infty} & \mu_{1-\alpha}(\omega) Z(\omega) \mathrm{e}^{-\omega t} \mathrm{~d} \omega \\
& =C_{3} \int_{0}^{\infty} \mu_{1-\alpha}(\omega) \mathrm{e}^{-\omega t} \mathrm{~d} \omega=C_{3} \frac{t^{-\alpha}}{\Gamma(1-\alpha)},
\end{aligned}
$$

which leads to algebraic decay of order $\alpha$ for the last term in eq. (60) for $t \rightarrow \infty$. For $\dot{\xi}$, we obtain the expression

$$
\begin{aligned}
\dot{\xi}(t)= & \sum_{j \text { odd }} 2 \frac{\mathrm{e}^{a_{j} t}}{f_{3}\left(r_{j}, \theta_{j}\right)}\left(\left(a_{j} f_{1}\left(r_{j}, \theta_{j}\right)\right.\right. \\
& \left.+b_{j} f_{2}\left(r_{j}, \theta_{j}\right)\right) \cos \left(b_{j} t\right) \\
& \left.+\left(a_{j} f_{2}\left(r_{j}, \theta_{j}\right)-b_{j} f_{1}\left(r_{j}, \theta_{j}\right)\right) \sin \left(b_{j} t\right)\right) \\
& -\int_{0}^{\infty} \mu_{1-\alpha}(\omega) Z(\omega) \omega \mathrm{e}^{-\omega t} \mathrm{~d} \omega,
\end{aligned}
$$

where the first terms again describe an exponentially decaying oscillation and the last term fulfills

$$
-\int_{0}^{\infty} \mu_{1-\alpha}(\omega) Z(\omega) \omega \mathrm{e}^{-\omega t} \mathrm{~d} \omega=-\alpha C_{3} \frac{t^{-\alpha-1}}{\Gamma(1-\alpha)},
$$

which again implies algebraic decay, this time of order $1+\alpha$ for $t \rightarrow \infty$. To conclude asymptotic stability of the trivial solution of eq. (17) from the asymptotic behavior of $\xi$, we still have to consider the last term in eq. (57). Therefore, we recall from eq. (8), that the initial infinite state $y(\omega, 0)$ has the form

$$
y(\omega, 0)=\int_{t_{0}}^{0} \mathrm{e}^{\omega \tau} \dot{q}(\tau) \mathrm{d} \tau
$$

which may be estimated as

$$
\begin{aligned}
|y(\omega, 0)| & =\left|\int_{t_{0}}^{0} \mathrm{e}^{\omega \tau} \dot{q}(\tau) \mathrm{d} \tau\right| \\
& =\left|\left[\mathrm{e}^{\omega \tau} q(\tau)\right]_{t_{0}}^{0}-\omega \int_{t_{0}}^{0} \mathrm{e}^{\omega \tau} q(\tau) \mathrm{d} \tau\right| \\
& \leq 2\|q\|_{\infty}+\omega \int_{-\infty}^{0} \mathrm{e}^{\omega \tau} \mathrm{d} \tau\|q\|_{\infty}=3\|q\|_{\infty},
\end{aligned}
$$

which is bounded if $q \in C B((-\infty, 0] ; \mathbb{R})$. Furthermore, consider the reformulation

$$
\int_{0}^{t} \mathrm{e}^{-\omega(t-\tau)} \dot{\xi}(\tau) \mathrm{d} \tau=\frac{\mathrm{d}}{\mathrm{d} t} \int_{0}^{t} \mathrm{e}^{-\omega(t-\tau)} \xi(\tau) \mathrm{d} \tau-\xi(0) \mathrm{e}^{-\omega t}
$$

of the inner integral in the last term of eq. (57). The last term in eq. (65) results in a term

$$
\left|\int_{0}^{\infty} \mu_{1-\alpha}(\omega) y(\omega, 0) \mathrm{e}^{-\omega t} \mathrm{~d} \omega\right| \leq 3\|q\|_{\infty} \frac{t^{-\alpha}}{\Gamma(1-\alpha)}
$$

in eq. (57). Substitution of the exponential terms of $\xi$ in the last term of eq. (57) using eq. (65) leads to the estimation

$$
\begin{aligned}
& \left|\int_{0}^{\infty} \mu_{1-\alpha}(\omega) y(\omega, 0) \frac{\mathrm{d}}{\mathrm{d} t} \int_{0}^{t} \mathrm{e}^{-\omega(t-\tau)} \mathrm{e}^{s_{j} \tau} \mathrm{d} \tau \mathrm{d} \omega\right| \\
& \leq 3\|q\|_{\infty}\left|\int_{0}^{\infty} \mu_{1-\alpha}(\omega) \frac{1}{\omega+s_{j}}\left(s_{j} \mathrm{e}^{s_{j} t}+\omega \mathrm{e}^{-\omega t}\right) \mathrm{d} \omega\right| \\
& \leq 3\|q\|_{\infty}\left(\left|s_{j} \int_{0}^{\infty} \frac{\mu_{1-\alpha}(\omega)}{\omega+s_{j}} \mathrm{~d} \omega\right| \mathrm{e}^{\operatorname{Re}\left(s_{j}\right) t}\right. \\
& \left.\quad+\int_{0}^{\infty} \mu_{1-\alpha}(\omega) \mathrm{e}^{-\omega t} \mathrm{~d} \omega\right) \\
& =3\|q\|_{\infty}\left(\left|s_{j}^{\alpha}\right| \mathrm{e}^{\operatorname{Re}\left(s_{j}\right) t}+\frac{t^{-\alpha}}{\Gamma(1-\alpha)}\right) .
\end{aligned}
$$

with roots $s_{j}$ of eq. (45). For the algebraic decay part in $\xi$ we obtain, again using the mean value theorem a constant $C_{4}>0$ and the term

$$
\begin{aligned}
& \mid \int_{0}^{\infty} \mu_{1-\alpha}(\omega) y(\omega, 0) \\
& \quad \times \frac{\mathrm{d}}{\mathrm{d} t} \int_{0}^{t} \mathrm{e}^{-\omega(t-\tau)} \int_{0}^{\infty} \mu_{1-\alpha}(\eta) Z(\eta) \mathrm{e}^{-\eta \tau} \mathrm{d} \eta \mathrm{d} \tau \mathrm{d} \omega \mid \\
& \leq C_{4}\left|\frac{\mathrm{d}}{\mathrm{d} t} \int_{0}^{\infty} \mu_{1-\alpha}(\omega) \int_{0}^{t} \mathrm{e}^{-\omega(t-\tau)} \frac{\tau^{-\alpha}}{\Gamma(1-\alpha)} \mathrm{d} \tau \mathrm{d} \omega\right| \\
& =C_{4}\left|\frac{\mathrm{d}}{\mathrm{d} t}{ }^{C} D_{0+}^{\alpha}\left(\frac{t^{1-\alpha}}{\Gamma(2-\alpha)}\right)\right| \\
& =C_{4}|1-2 \alpha| \frac{t^{-2 \alpha}}{\Gamma(2-2 \alpha)} .
\end{aligned}
$$

in the last term of eq. (57). In summary, we obtain sufficient conditions (49) and (50) for global asymptotic stability of the equilibrium of eq. (17), from which we retrieve a Lyapunov functional in Section 4.5.2.

Remark 4.9. It is even possible to obtain a purely exponential solution of eq. (17) without algebraic decay. Choose the initial function $q(\tau)=\mathrm{e}^{s_{j} \tau}$ for $\tau \in(-\infty, 0]$ (which is not in $C B((-\infty, 0] ; \mathbb{R}))$ for a root $s_{j}$ of eq. (45). This leads to initial conditions 


$$
\dot{q}(\tau)=s_{j} \mathrm{e}^{s_{j} t}, \quad y(\omega, 0)=\int_{-\infty}^{0} \mathrm{e}^{\omega \tau} s_{j} \mathrm{e}^{s_{j} \tau} \mathrm{d} \tau=\frac{s_{j}}{\omega+s_{j}} .
$$

Using this function in the Laplace transform (55) leads to

$$
\begin{aligned}
Q(s)= & \frac{m s+d+c s^{\alpha-1}}{m s^{2}+d s+c s^{\alpha}+k}+\frac{m s_{j}}{m s^{2}+d s+c s^{\alpha}+k} \\
& -\frac{c s_{j}}{m s^{2}+d s+c s^{\alpha}+k} \int_{0}^{\infty} \frac{\mu_{1-\alpha}(\omega)}{(\omega+s)\left(\omega+s_{j}\right)} \mathrm{d} \omega \\
= & \frac{m s+d+c s^{\alpha-1}+m s_{j}}{m s^{2}+d s+c s^{\alpha}+k} \\
& -c s_{j} \frac{\int_{0}^{\infty} \frac{\mu_{1-\alpha}(\omega)}{\omega+s_{j}} \mathrm{~d} \omega-\int_{0}^{\infty} \frac{\mu_{1-\alpha}(\omega)}{\omega+s} \mathrm{~d} \omega}{\left(s-s_{j}\right)\left(m s^{2}+d s+c s^{\alpha}+k\right)} \\
= & \frac{\left(m s+d+c s^{\alpha-1}+m s_{j}\right)\left(s-s_{j}\right)}{\left(s-s_{j}\right)\left(m s^{2}+d s+c s^{\alpha}+k\right)} \\
& -c s_{j} \frac{s_{j}^{\alpha-1}-s^{\alpha-1}}{\left(s-s_{j}\right)\left(m s^{2}+d s+c s^{\alpha}+k\right)} \\
= & \frac{1}{s-s_{j}} .
\end{aligned}
$$

Hence, the solution is

$$
q(t)=\mathrm{e}^{s_{j} t}, \quad \forall t,
$$

which shows that the integral term in eq. (42) should in general not be omitted.

\subsubsection{Lyapunov functional}

To formulate a Lyapunov functional, we will use the identities of the next proposition.

Proposition 4.10. For $\alpha \in(0,1)$ and $r>0$, the identities

$$
\begin{gathered}
\int_{0}^{\infty} \frac{\mu_{1-\alpha}(\omega)}{\omega^{2}+r^{2}} \mathrm{~d} \omega=\cos \left(\frac{\alpha \pi}{2}\right) r^{\alpha-2} \\
\int_{0}^{\infty} \frac{\mu_{1-\alpha}(\omega) \omega}{\omega^{2}+r^{2}} \mathrm{~d} \omega=\sin \left(\frac{\alpha \pi}{2}\right) r^{\alpha-1}
\end{gathered}
$$

hold.

Proof. Substitute $\eta=\omega^{2}$ and $\mathrm{d} \eta=2 \omega \mathrm{d} \omega$ in the integral and obtain

$$
\begin{aligned}
\int_{0}^{\infty} \frac{\mu_{1-\alpha}(\omega)}{\omega^{2}+r^{2}} \mathrm{~d} \omega & =\frac{\sin (\alpha \pi)}{\pi} \int_{0}^{\infty} \frac{\omega^{\alpha-1}}{\omega^{2}+r^{2}} \mathrm{~d} \omega \\
& =\frac{\sin (\alpha \pi)}{2 \pi} \int_{0}^{\infty} \frac{\eta^{\frac{\alpha}{2}-1}}{\eta+r^{2}} \mathrm{~d} \eta \\
& =\frac{\sin (\alpha \pi)}{2 \sin \left(\frac{\alpha \pi}{2}\right)} \int_{0}^{\infty} \frac{\mu_{1-\frac{\alpha}{2}}(\eta)}{\eta+r^{2}} \mathrm{~d} \eta
\end{aligned}
$$

Using sine-double-angle formula and eq. (41), we directly obtain eq. (66). The proof of eq. (67) is analogous.

In the following, let $r_{*}$ be the solution of the first equation of (47). We reformulate eq. (17) using eqs. (7), (36) and Proposition 4.10 as

$$
\begin{aligned}
m \ddot{q}(t)= & -k q(t)-d \dot{q}(t)-c r_{*}^{2} \int_{0}^{\infty} \frac{\mu_{1-\alpha}(\omega)}{\omega^{2}+r_{*}^{2}} y(\omega, t) \mathrm{d} \omega \\
& -c \int_{0}^{\infty} \frac{\mu_{1-\alpha}(\omega) \omega}{\omega^{2}+r_{*}^{2}} \omega y(\omega, t) \mathrm{d} \omega \\
= & -\left(k+c \cos \left(\frac{\alpha \pi}{2}\right) r_{*}^{\alpha}\right) q(t) \\
& -\left(d+c \sin \left(\frac{\alpha \pi}{2}\right) r_{*}^{\alpha-1}\right) \dot{q}(t) \\
& +c r_{*}^{2} \int_{0}^{\infty} \frac{\mu_{1-\alpha}(\omega)}{\omega^{2}+r_{*}^{2}} \omega Y(\omega, t) \mathrm{d} \omega \\
& +c \int_{0}^{\infty} \frac{\mu_{1-\alpha}(\omega)}{\omega^{2}+r_{*}^{2}} \omega \dot{y}(\omega, t) \mathrm{d} \omega .
\end{aligned}
$$

Introducing the quantities

$$
\tilde{k}=k+c \cos \left(\frac{\alpha \pi}{2}\right) r_{*}^{\alpha}, \quad \tilde{d}=d+c \sin \left(\frac{\alpha \pi}{2}\right) r_{*}^{\alpha-1},
$$

and new coordinates

$$
\begin{aligned}
\tilde{q}(t) & =q(t)-\frac{c}{\tilde{k}} r_{*}^{2} \int_{0}^{\infty} \frac{\mu_{1-\alpha}(\omega)}{\omega^{2}+r_{*}^{2}} \omega Y(\omega, t) \mathrm{d} \omega \\
& =\frac{k}{\tilde{k}} q(t)+\frac{c}{\tilde{k}} r_{*}^{2} \int_{0}^{\infty} \frac{\mu_{1-\alpha}(\omega)}{\omega^{2}+r_{*}^{2}} y(\omega, t) \mathrm{d} \omega
\end{aligned}
$$

and

$$
\tilde{v}(t)=\dot{q}(t)-\frac{c}{m} \int_{0}^{\infty} \frac{\mu_{1-\alpha}(\omega)}{\omega^{2}+r_{*}^{2}} \omega y(\omega, t) \mathrm{d} \omega
$$

we obtain the system

$$
\left\{\begin{array}{l}
\dot{\tilde{q}}(t)=\tilde{v}(t), \\
\dot{\tilde{v}}(t)=-\frac{\tilde{k}}{m} \tilde{q}(t)-\frac{\tilde{d}}{m} \tilde{v}(t)-\frac{\tilde{d} c}{m^{2}} \int_{0}^{\infty} \frac{\mu_{1-\alpha}(\omega)}{\omega^{2}+r_{*}^{2}} \omega y(\omega, t) \mathrm{d} \omega .
\end{array}\right.
$$

Note that the first equation in (72) holds, as $r_{*}$ is a solution of the first equation of (47). Moreover, we consider the candidate Lyapunov functional

$$
\begin{aligned}
V_{3}\left(t, q_{t}, v_{t}\right)= & \frac{m}{2} \tilde{v}_{t}^{2}(0)+\frac{\tilde{k}}{2} \tilde{q}_{t}^{2}(0) \\
& +\frac{\tilde{d} c}{2 m} \int_{0}^{\infty} \frac{\mu_{1-\alpha}(\omega)}{\omega^{2}+r_{*}^{2}} \omega y^{2}(\omega, t) \mathrm{d} \omega
\end{aligned}
$$

and prove inequality (26) for $V_{3}$ w.r.t. the functions $q_{t}$ and $v_{t}$. Therefore, consider the split of the integral term in eq. (73) 


$$
\begin{aligned}
\int_{0}^{\infty} \frac{\mu_{1-\alpha}(\omega)}{\omega^{2}+r_{*}^{2}} \omega y^{2}(\omega, t) \mathrm{d} \omega \\
=\int_{0}^{1} \frac{\mu_{1-\alpha}(\omega)}{\omega^{2}+r_{*}^{2}} \omega y^{2}(\omega, t) \mathrm{d} \omega \\
\quad+\int_{1}^{\infty} \frac{\mu_{1-\alpha}(\omega)}{\omega^{2}+r_{*}^{2}} \omega y^{2}(\omega, t) \mathrm{d} \omega
\end{aligned}
$$

and use the mean value theorem for the first term and the inequality $\omega \geq 1$ in the second term to find a constant $\tilde{C}>0$, such that

$$
\begin{aligned}
& \int_{0}^{\infty} \frac{\mu_{1-\alpha}(\omega)}{\omega^{2}+r_{*}^{2}} \omega y^{2}(\omega, t) \mathrm{d} \omega \\
& \quad \geq \tilde{C} \int_{0}^{\infty} \frac{\mu_{1-\alpha}(\omega)}{\omega^{2}+r_{*}^{2}} y^{2}(\omega, t) \mathrm{d} \omega
\end{aligned}
$$

Moreover, we use Hölder's inequality to obtain

$$
\begin{aligned}
& \left(\int_{0}^{\infty} \frac{\mu_{1-\alpha}(\omega)}{\omega^{2}+r_{*}^{2}} y(\omega, t) \mathrm{d} \omega\right)^{2} \\
& \leq \int_{0}^{\infty} \frac{\mu_{1-\alpha}(\omega)}{\omega^{2}+r_{*}^{2}} \mathrm{~d} \omega \cdot \int_{0}^{\infty} \frac{\mu_{1-\alpha}(\omega)}{\omega^{2}+r_{*}^{2}} y^{2}(\omega, t) \mathrm{d} \omega
\end{aligned}
$$

and

$$
\begin{aligned}
& \left(\int_{0}^{\infty} \frac{\mu_{1-\alpha}(\omega)}{\omega^{2}+r_{*}^{2}} \omega y(\omega, t) \mathrm{d} \omega\right)^{2} \\
& \leq \int_{0}^{\infty} \frac{\mu_{1-\alpha}(\omega) \omega}{\omega^{2}+r_{*}^{2}} \mathrm{~d} \omega \cdot \int_{0}^{\infty} \frac{\mu_{1-\alpha}(\omega)}{\omega^{2}+r_{*}^{2}} \omega y^{2}(\omega, t) \mathrm{d} \omega .
\end{aligned}
$$

Using the three inequalities above and Proposition 4.10, we can estimate (73) as

$$
\begin{aligned}
V_{3}\left(t, q_{t}, v_{t}\right) \geq & \frac{m}{2} \tilde{v}_{t}^{2}(0)+\frac{\tilde{d} c}{4 m \sin \left(\frac{\alpha \pi}{2}\right) r_{*}^{\alpha-1}} \\
& \times\left(\int_{0}^{\infty} \frac{\mu_{1-\alpha}(\omega)}{\omega^{2}+r_{*}^{2}} \omega y(\omega, t) \mathrm{d} \omega\right)^{2} \\
+ & \frac{\tilde{k}}{2} \tilde{q}_{t}^{2}(0)+\frac{\tilde{d} c \tilde{C}}{4 m \cos \left(\frac{\alpha \pi}{2}\right) r_{*}^{\alpha-2}} \\
& \times\left(\int_{0}^{\infty} \frac{\mu_{1-\alpha}(\omega)}{\omega^{2}+r_{*}^{2}} y(\omega, t) \mathrm{d} \omega\right)^{2}
\end{aligned}
$$

Finally, applying the general relation

$$
(a+b)^{2}+\gamma b^{2}=\frac{\gamma}{1+\gamma} a^{2}+\left(\frac{a}{\sqrt{1+\gamma}}+\sqrt{1+\gamma} b\right)^{2}
$$

for $a, b, \gamma \in \mathbb{R}, \gamma>0$ on the first two and the last two terms of (77) using eqs. (70) and (71), we obtain inequality (26) for $V_{3}$. Furthermore, we compute the rate of $V_{3}$ as

$$
\begin{aligned}
\dot{V}_{3}= & m \dot{\tilde{v}}_{t}(0) \tilde{v}_{t}(0)+\tilde{k} \tilde{q}_{t}(0) \dot{\tilde{q}}_{t}(0) \\
& +\frac{\tilde{d} c}{m} \int_{0}^{\infty} \frac{\mu_{1-\alpha}(\omega)}{\omega^{2}+r_{*}^{2}} \omega y(\omega, t) \dot{y}(\omega, t) \mathrm{d} \omega .
\end{aligned}
$$

Inserting the dynamics from (72), we obtain

$$
\begin{aligned}
\dot{V}_{3}= & -\tilde{d} \tilde{v}^{2}(t)+\frac{\tilde{d} c^{2}}{m^{2}}\left(\int_{0}^{\infty} \frac{\mu_{1-\alpha}(\omega)}{\omega^{2}+r_{*}^{2}} \omega y(\omega, t) \mathrm{d} \omega\right)^{2} \\
& -\frac{\tilde{d} c}{m} \int_{0}^{\infty} \frac{\mu_{1-\alpha}(\omega)}{\omega^{2}+r_{*}^{2}} \omega^{2} y^{2}(\omega, t) \mathrm{d} \omega .
\end{aligned}
$$

Again, using Hölder's inequality leads to

$$
\begin{aligned}
& \left(\int_{0}^{\infty} \frac{\mu_{1-\alpha}(\omega)}{\omega^{2}+r_{*}^{2}} \omega y(\omega, t) \mathrm{d} \omega\right)^{2} \\
& \leq \int_{0}^{\infty} \frac{\mu_{1-\alpha}(\omega)}{\omega^{2}+r_{*}^{2}} \mathrm{~d} \omega \cdot \int_{0}^{\infty} \frac{\mu_{1-\alpha}(\omega)}{\omega^{2}+r_{*}^{2}} \omega^{2} y^{2}(\omega, t) \mathrm{d} \omega
\end{aligned}
$$

and together with eq. (66), we finally obtain

$$
\begin{aligned}
\dot{V}_{3} \leq-\tilde{d} \tilde{v}^{2}(t)- & \frac{\tilde{d} c}{m}\left(1-\frac{c}{m} \cos \left(\frac{\alpha \pi}{2}\right) r_{*}^{\alpha-2}\right) \\
& \times \int_{0}^{\infty} \frac{\mu_{1-\alpha}(\omega)}{\omega^{2}+r_{*}^{2}} \omega^{2} y^{2}(\omega, t) \mathrm{d} \omega
\end{aligned}
$$

where, due to eq. (47)

$$
\begin{array}{r}
1-\frac{c}{m} \cos \left(\frac{\alpha \pi}{2}\right) r_{*}^{\alpha-2}>0 \\
\Leftrightarrow m r_{*}^{2}-c \cos \left(\frac{\alpha \pi}{2}\right) r_{*}^{\alpha}=k>0 .
\end{array}
$$

In summary, we found a Lyapunov functional $V_{3}$, such that $\dot{V}_{3} \leq 0$, which has the form of an energy functional w.r.t. the new coordinates $\tilde{q}_{t}$ and $\tilde{v}_{t}$ but it has the disadvantage that we cannot prove asymptotic stability of the trivial solution of eq. (17) with the help of $V_{3}$ as in the classical case of the damped linear oscillator using the total mechanical energy as a Lyapunov function. Hence, we introduce another Lyapunov functional $V_{4}$, which is related to the functional $V_{2}$ in Section 4.4 , to prove the following proposition.

Proposition 4.11. Let $m, k, c>0, \alpha \in(0,1)$ and let $r=r_{*}>0$ be the solution of

$$
-m r^{2}+c r^{\alpha} \cos \left(\frac{\alpha \pi}{2}\right)+k=0
$$

Let $d \in \mathbb{R}$ be such that the inequality

$$
d r_{*}+c r_{*}^{\alpha} \sin \left(\frac{\alpha \pi}{2}\right)>0
$$

holds. Then the trivial solution of eq. (17) is asymptotically stable. 
Proof. We introduce the Lyapunov functional

$$
\begin{aligned}
V_{4}\left(t, q_{t}, v_{t}\right)= & \frac{1}{2} m \tilde{v}_{t}^{2}(0)+\frac{1}{2} \tilde{k} \tilde{q}_{t}^{2}(0)+\frac{\tilde{d}^{2}}{4 m} \tilde{q}_{t}^{2}(0) \\
& +\frac{1}{2} \tilde{d} \tilde{q}_{t}(0) \tilde{v}_{t}(0) \\
& +\frac{\tilde{d} c}{2 m} \int_{0}^{\infty} \frac{\mu_{1-\alpha}(\omega)}{\omega^{2}+r_{*}^{2}} \omega y^{2}(\omega, t) \mathrm{d} \omega \\
& +\frac{\tilde{d}^{2} c}{4 m^{2}} \int_{0}^{\infty} \frac{\mu_{1-\alpha}(\omega)}{\omega^{2}+r_{*}^{2}} \omega^{2} Y^{2}(\omega, t) \mathrm{d} \omega \\
& -\frac{\tilde{d}^{2} c}{4 m^{2}} \frac{c}{\tilde{k}} r_{*}^{2}\left(\int_{0}^{\infty} \frac{\mu_{1-\alpha}(\omega)}{\omega^{2}+r_{*}^{2}} \omega Y(\omega, t) \mathrm{d} \omega\right)^{2}
\end{aligned}
$$

and check the conditions in Theorem 4.3. Once again, we use Hölder's inequality to obtain

$$
\begin{aligned}
& \left(\int_{0}^{\infty} \frac{\mu_{1-\alpha}(\omega)}{\omega^{2}+r_{*}^{2}} \omega Y(\omega, t) \mathrm{d} \omega\right)^{2} \\
& \leq \int_{0}^{\infty} \frac{\mu_{1-\alpha}(\omega)}{\omega^{2}+r_{*}^{2}} \mathrm{~d} \omega \cdot \int_{0}^{\infty} \frac{\mu_{1-\alpha}(\omega)}{\omega^{2}+r_{*}^{2}} \omega^{2} Y^{2}(\omega, t) \mathrm{d} \omega .
\end{aligned}
$$

Together with (76), estimation (38) and Proposition 4.10 we obtain

$$
\begin{aligned}
& V_{4}\left(t, q_{t}, v_{t}\right) \\
& \qquad \frac{1}{4} m \tilde{v}_{t}^{2}(0)+\frac{1}{2} \tilde{k} \tilde{q}_{t}^{2}(0) \\
& \quad+\frac{\tilde{d} c}{2 m \sin \left(\frac{\alpha \pi}{2}\right) r_{*}^{\alpha-1}}\left(\int_{0}^{\infty} \frac{\mu_{1-\alpha}(\omega)}{\omega^{2}+r_{*}^{2}} \omega y(\omega, t) \mathrm{d} \omega\right)^{2} \\
& \quad+\frac{\tilde{d}^{2} c}{4 m^{2}} \frac{c}{\tilde{k}} r_{*}^{2}\left(\frac{\tilde{k}}{c \cos \left(\frac{\alpha \pi}{2}\right) r_{*}^{\alpha}}-1\right) \\
& \quad \times\left(\int_{0}^{\infty} \frac{\mu_{1-\alpha}(\omega)}{\omega^{2}+r_{*}^{2}} \omega Y(\omega, t) \mathrm{d} \omega\right)^{2} .
\end{aligned}
$$

All coefficients in this estimation are positive and, again using eqs. (70), (71) and (78), we obtain relation (26) for $V_{4}$. Furthermore, we compute the rate of $V_{4}$ as

$$
\begin{aligned}
\dot{V}_{4}= & m \tilde{v}_{t}(0) \dot{\tilde{v}}_{t}(0)+\tilde{k} \tilde{q}_{t}(0) \dot{\tilde{q}}_{t}(0)+\frac{\tilde{d}}{2} \tilde{v}_{t}(0) \dot{\tilde{q}}_{t}(0) \\
& +\frac{\tilde{d}_{2}}{2} \tilde{q}_{t}(0) \dot{\tilde{v}}_{t}(0)+\frac{\tilde{d}^{2}}{2 m} \tilde{q}_{t}(0) \dot{\tilde{q}}_{t}(0) \\
& +\frac{\tilde{d} c}{m} \int_{0}^{\infty} \frac{\mu_{1-\alpha}(\omega)}{\omega^{2}+r_{*}^{2}} \omega y(\omega, t) \dot{y}(\omega, t) \mathrm{d} \omega \\
& +\frac{\tilde{d}^{2} c}{2 m^{2}} \int_{0}^{\infty} \frac{\mu_{1-\alpha}(\omega)}{\omega^{2}+r_{*}^{2}} \omega Y(\omega, t) \omega \dot{Y}(\omega, t) \mathrm{d} \omega
\end{aligned}
$$

$$
\begin{gathered}
-\frac{\tilde{d}^{2} c}{2 m^{2}} \frac{c}{\tilde{k}} r_{*}^{2}\left(\int_{0}^{\infty} \frac{\mu_{1-\alpha}(\omega)}{\omega^{2}+r_{*}^{2}} \omega Y(\omega, t) \mathrm{d} \omega\right) \\
\quad \times\left(\int_{0}^{\infty} \frac{\mu_{1-\alpha}(\omega)}{\omega^{2}+r_{*}^{2}} \omega \dot{Y}(\omega, t) \mathrm{d} \omega\right)
\end{gathered}
$$

Inserting the dynamics and using eqs. (81) and (74), we obtain

$$
\begin{aligned}
\dot{V}_{4} \leq & -\frac{\tilde{d}}{2} \tilde{v}^{2}(t)-\frac{\tilde{d} c}{m}\left(1-\frac{c}{m} \cos \left(\frac{\alpha \pi}{2}\right) r_{*}^{\alpha-2}\right) \\
& \times \int_{0}^{\infty} \frac{\mu_{1-\alpha}(\omega)}{\omega^{2}+r_{*}^{2}} \omega^{2} y^{2}(\omega, t) \mathrm{d} \omega \\
& -\frac{\tilde{k} \tilde{d}}{2 m} \tilde{q}^{2}(t)-\frac{\tilde{d}^{2} c \tilde{C}}{2 m^{2}} \int_{0}^{\infty} \frac{\mu_{1-\alpha}(\omega)}{\omega^{2}+r_{*}^{2}} y^{2}(\omega, t) \mathrm{d} \omega .
\end{aligned}
$$

Again, with the help of the estimations (75), (81) and eqs. (70), (71) and (78), one can prove that $\dot{V}_{4}$ fulfills inequality (29) w.r.t. $q$ and $v$. This completes the proof.

\section{Remark 4.12.}

(a) As a special case, Proposition 4.11 proves asymptotic stability of the trivial solution of eq. (17) for the case $d=0$. Previously in Section 4.3, with the help of the energy functional we could only prove stability but not attractivity of the trivial solution.

(b) The conditions for asymptotic stability in Proposition 4.11 are equivalent to the necessary and sufficient conditions obtained by the eigenvalue analysis in Proposition 4.7. In that sense, the choice of the functionals $V_{3}$ and $V_{4}$ is optimal.

\subsubsection{Example: Stick-slip oscillator [29-31]}

In this section, we describe a model of a mechanical system, where effective negative linear damping occurs in the linearization of the equation of motion around an equilibrium and we give sufficient conditions for local asymptotic stability of the equilibrium using Proposition 4.11. Consider a mass $m$ suspended by a spring with spring coefficient $k$, and a springpot with coefficient $c$ and differentiation order $\alpha \in(0,1)$, which is sliding on a conveyor belt as in Figure 7. By $q$ we denote the displacement of the mass. The belt moves with a constant velocity $v_{\mathrm{dr}}>0$ in the direction of $q$ and we assume friction between the mass and the belt, which leads to a friction force $F_{T}$, such that the equation of motion reads as

$$
m \ddot{q}(t)=F_{T}-c^{C} D_{t_{0}+}^{\alpha} q(t)-k q(t) .
$$



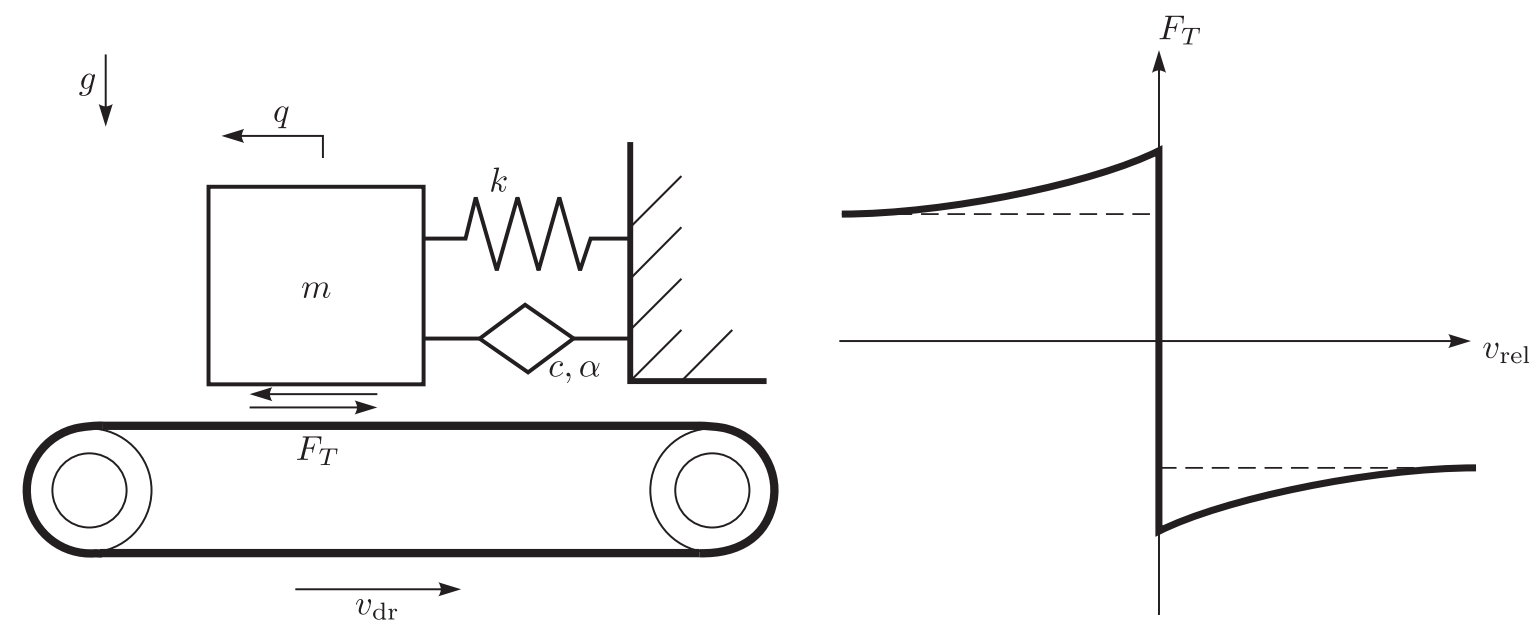

Figure 7: Fractionally damped oscillator with dry friction and graph of a set-valued force law describing the Stribeck effect.

For the friction force $F_{T}$ in the slip phase we consider the force law

$$
F_{T}=-\mu\left(v_{\text {rel }}\right) F_{N} \operatorname{sign}\left(v_{\text {rel }}\right), \quad v_{\text {rel }} \neq 0
$$

where

$$
v_{\text {rel }}(t)=\dot{q}(t)-v_{\mathrm{dr}}
$$

is the relative velocity between mass and belt,

$$
F_{N}=m g
$$

is the normal force acting on the mass and $\mu=\mu(v)$ is the friction coefficient depending on the relative velocity, where the function $\mu$ increases (at least) for small negative values of $v_{\text {rel }}$, i.e.

$$
\mu^{\prime}(-|v|)>0, \quad|v| \ll 1
$$

which is known as the Stribeck effect. We consider the slip equilibrium $q^{*}$ of eq. (84)

$$
\begin{aligned}
0 & =F_{T}-k q^{*}, \\
-F_{T} & =\mu\left(-v_{\mathrm{dr}}\right) F_{N} \operatorname{sign}\left(-v_{\mathrm{dr}}\right)=-\mu\left(-v_{\mathrm{dr}}\right) m g,
\end{aligned}
$$

which implies

$$
q^{*}=\frac{\mu\left(-v_{\mathrm{dr}}\right) m g}{k}
$$

We introduce a new coordinate

$$
\bar{q}=q-q^{*}
$$

such that we reformulate eq. (84) in terms of $\bar{q}$ as

$$
\begin{aligned}
& m \ddot{\bar{q}}(t)+c^{C} D_{t_{0}+}^{\alpha} \bar{q}(t)+k\left(\bar{q}(t)+q^{*}\right) \\
& \quad=-\mu\left(\dot{\bar{q}}(t)-v_{\mathrm{dr}}\right) m g \operatorname{sign}\left(\dot{\bar{q}}(t)-v_{\mathrm{dr}}\right) .
\end{aligned}
$$

Linearizing the right-hand side of eq. (93) near the equilibrium leads to

$$
\begin{aligned}
& m \ddot{\bar{q}}(t)+c^{C} D_{t_{0^{+}}}^{\alpha} \bar{q}(t)+k\left(\bar{q}(t)+q^{*}\right) \\
& \quad=\mu\left(-v_{\mathrm{dr}}\right) m g+\mu^{\prime}\left(-v_{\mathrm{dr}}\right) m g \dot{\bar{q}}(t)+\mathcal{O}\left(\dot{\bar{q}}^{2}\right),
\end{aligned}
$$

which together with eq. (91) leads to the linearized equation

$$
m \ddot{\bar{q}}(t)-\mu^{\prime}\left(-v_{\mathrm{dr}}\right) m g \dot{\bar{q}}(t)+c^{C} D_{t_{0^{+}}}^{\alpha} \bar{q}(t)+k \bar{q}(t)=\mathcal{O}\left(\dot{\bar{q}}^{2}\right)
$$

Hence, using Proposition 4.11, we obtain the condition

$$
c r_{*}^{\alpha-1} \sin \left(\frac{\alpha \pi}{2}\right)>\mu^{\prime}\left(-v_{\mathrm{dr}}\right) m g
$$

for local asymptotic stability of the slip equilibrium $q^{*}$, where again $r_{*}$ is the solution of

$$
-m r^{2}+c r^{\alpha} \cos \left(\frac{\alpha \pi}{2}\right)+k=0 .
$$

\section{Conclusion}

The previous sections have given a complete stability analysis of a linear single degree-of-freedom mass-springdashpot-springpot system, including an eigenvalue analysis, a derivation of total mechanical energy and Lyapunov functionals for various cases. The major merit of the paper lies in the extension of the Lyapunov stability framework to fractionally damped mechanical systems as this step is essential for rigorous proofs of global stability properties of nonlinear systems and further bifurcation 
analysis. Specifically, the results in Section 4 show how the terms in the Lyapunov functional related to the springpot element have to be split, i. e. how one has to deal with fractional damping in a Lyapunov setting.

The scope has been limited to a single degree-offreedom oscillator with viscous and fractional damping. Obviously, these results need to be generalized to multi degree-of-freedom systems. That such a generalization is possible, at least in special cases, can be seen by looking at a linear multi degree-of-freedom system

$$
M \ddot{q}+D_{v} \dot{q}+D_{f} D_{t_{0}+}^{\alpha} q+K q=0
$$

with symmetric system matrices. If the viscous damping matrix $D_{v}$ and fractional damping matrix $D_{f}$ are proportional to the mass and stiffness matrices (or, more generally, Caughey damping), then one can use known results from linear vibration analysis to show that the modal equations are decoupled single degree-of-freedom fractionally damped oscillators as dealt with here. Clearly, further research is needed for more general cases.

A key result of the paper is the Lyapunov stability condition for systems having negative viscous damping but a sufficient amount of positive fractional damping. The example of a stick-slip oscillator demonstrates that negative viscous damping is relevant. More generally, all Hopf bifurcation instabilities in non-conservative mechanical systems are due to negative effective viscous damping. The mechanism leading to effective negative damping may be quite complicated, e.g. be caused by mode coupling, follower forces or aerodynamic forces (flutter), and also be attributed to Ziegler's paradox. A typical task within control theory is the design of a feedback law which stabilizes the equilibrium and a common tool to achieve this is Lyapunov-based control design. Hence, Lyapunov methods can be used to stabilize systems with negative effective viscous damping, either with integer or fractional PID control. The Lyapunov techniques developed here, specifically the results on antidamping, may prove to be instrumental for this purpose.

Funding: This work was supported by the Federal Ministry of Education and Research of Germany (BMBF) (Funder Id: http://dx.doi.org/10.13039/501100002347, Grant Number: 01IS17096B).

\section{References}

[1] K. Diethelm, The analysis of fractional differential equations: An application-oriented exposition using differential operators of Caputo type, Lecture notes in mathematics, Springer, Berlin, 2010.
[2] I. Podlubny, Fractional differential equations. An introduction to fractional derivatives, fractional differential equations, to methods of their solution and some of their applications, Mathematics in science and engineering Vol. 198, Academic Press, San Diego, 1999, XXIV.

[3] H. Sun, Y. Zhang, D. Baleanu, W. Chen and Y. Chen, A new collection of real world applications of fractional calculus in science and engineering, Commun. Nonlinear Sci. Numer. Simul. 64 (2018), 213-231.

[4] R. L. Bagley and P. J. Torvik, Fractional calculus in the transient analysis of viscoelastically damped structures, AIAA J. 23 (1985), 918-925.

[5] A. Schmidt and L. Gaul, Finite element formulation of viscoelastic constitutive equations using fractional time derivatives, Nonlinear Dyn. 29 (2002), 37-55.

[6] P. Shah and S. Agashe, Review of fractional PID controller, Mechatronics 38 (2016), 29-41.

[7] H. K. Khalil, Nonlinear systems, 3. ed., Prentice Hall, Upper Saddle River, 2002.

[8] T. A. Burton, Volterra integral and differential equations, Mathematics in science and engineering Vol. 202, Amsterdam, Elsevier, 2005.

[9] T. A. Burton, Stability and periodic solutions of ordinary and functional differential equations, Mathematics in science and engineering Vol. 178, Academic Press, Orlando, 1985.

[10] J. K. Hale, Theory of functional differential equations, 2 ed, Applied mathematical sciences Vol. 3, Springer, New York Heidelberg Berlin, 1977.

[11] V. B. Kolmanovskii and V. R. Nosov, Stability of functional differential equations, Mathematics in science and engineering Vol. 180, Academic Press, London, 1986.

[12] J. P. LaSalle and Z. Artstein, The stability of dynamical systems, Regional Conference Series in Applied Mathematics, vol. 25, Society for Industrial and Applied Mathematics, Philadelphia, 1976.

[13] J.-C. Trigeassou, N. Maamri and A. Oustaloup, Lyapunov stability of commensurate fractional order systems: A physical interpretation, J. Comput. Nonlinear Dyn. 11 (2016), 051007.

[14] J.-C. Trigeassou, N. Maamri, J. Sabatier and A. Oustaloup, A Lyapunov approach to the stability of fractional differential equations, Signal Process. 91 (2011), 437-445.

[15] M. Hinze, A. Schmidt and R. I. Leine, Mechanical representation and stability of dynamical systems containing fractional springpot elements, Proceedings of the IDETC Quebec, Canada, 2018.

[16] K. D. Papoulia, V. P. Panoskaltsis, N. V. Kurup and I. Korovajchuk, Rheological representation of fractional order viscoelastic material models, Rheologica Acta 49 (2010), 381-400.

[17] H. Schiessel and A. Blumen, Hierarchical analogues to fractional relaxation equations, J Phys. A Math. Gen. 26 (1993), 5057-5069.

[18] G. Montseny, Diffusive representation of pseudo-differential time-operators, ESAIM: Proc. 5 (1998), 159-175.

[19] D. Matignon, Stability properties for generalized fractional differential systems, ESAIM: Proc. 5 (1998), 145-158.

[20] J. Trigeassou, N. Maamri, J. Sabatier and A. Oustaloup, State variables and transients of fractional order differential systems, Comput. Math. Appl. 64 (2012), 3117-3140. Advances in FDE, III. 
[21] J.-C. Trigeassou, N. Maamri, J. Sabatier and A. Oustaloup, Transients of fractional-order integrator and derivatives, Signal Image Video Process. 6(3) (2012), 359-372.

[22] D. Matignon, Stability results for fractional differential equations with applications to control processing, Computational Engineering in Systems Applications Multiconference, IMACS, IEEE-SMC, Lille, France (1996), 963-968.

[23] J. Sabatier, M. Moze and C. Farges, LMI stability conditions for fractional order systems, Comput. Math. Appl. 59 (2010), 1594-1609.

[24] R. Agarwal, D. O'Regan and S. Hristova, Stability of Caputo fractional differential equations by Lyapunov functions, Appl. Math. 60 (2015), 653-676.

[25] M. A. Duarte-Mermoud, N. Aguila-Camacho, J. A. Gallegos and R. Castro-Linares, Using general quadratic Lyapunov functions to prove Lyapunov uniform stability for fractional order systems, Commun. Nonlinear Sci. Numer. Simul. 22 (2015), 650-659.
[26] Y. Li, Y. Q. Chen and I. Podlubny, Stability of fractional-order nonlinear dynamic systems: Lyapunov direct method and generalized Mittag-Leffler stability, Comput. Math. Appl. 59 (2010), 1810-1821.

[27] L.-L. Liu and J.-S. Duan, A detailed analysis for the fundamental solution of fractional vibration equation, Open Math. 13 (2015), 826-838.

[28] M. Naber, Linear fractionally damped oscillator, Int. J. Diff. Eq. 2010 (2010), 1-12, Article ID: 197020.

[29] U. Galvanetto, S. R. Bishop and L. Briseghella, Mechanical stick-slip vibrations, Int. J. Bifurcation Chaos 5 (1995), 637-651.

[30] R. A. Ibrahim, Friction-induced vibration, chatter, squeal, and chaos; Part I: Mechanics of contact and friction, ASME Appl. Mech. Rev. 47 (1994), 209-226.

[31] R. I. Leine and H. Nijmeijer, Dynamics and bifurcations of non-smooth mechanical systems, Lecture notes in applied and computational mechanics Vol. 18, Springer, Berlin Heidelberg New York, 2004, XII. 